\title{
Efficient Unsteady Model Estimation Using Computational and Experimental Data
}

\author{
Patrick C. Murphy, ${ }^{1}$ Neal Frink, ${ }^{2}$ S. Naomi McMillin, ${ }^{3}$ Kevin Cunningham, ${ }^{4}$ and Gautam H. Shah ${ }^{5}$ \\ NASA Langley Research Center, Hampton, VA 23681
}

\begin{abstract}
Improving aircraft simulations for pilot training in loss-of-control and stalled conditions is one goal of NASA research in the System Wide Safety Program. One part of this effort is to develop appropriate generic aerodynamic models that provide representative responses in simulation for a given class of aircraft. In this part of the flight envelope nonlinear unsteady responses are often present and may require an extended aerodynamic model compared to that used in the conventional flight envelope. In this preliminary study, two objectives are addressed. First, to obtain a representative model for a NASA generic aircraft at an unsteady condition in the flight envelope and second, to evaluate the techniques involved. To meet these objectives, two different generic aircraft configurations are modeled using both experimental and analytical data. With these results, an initial assessment of the efficiency and quality of the tools and test techniques are evaluated to develop guidance for analytical and experimental approaches to unsteady modeling.
\end{abstract}

\section{Nomenclature}

\begin{tabular}{|c|c|c|c|}
\hline$A_{j}, B_{j}$ & $=$ Fourier coefficients & $t$ & $=$ time, sec \\
\hline$a, b_{1}$ & $=$ deficiency function parameters & $V$ & $=$ velocity, fps \\
\hline$b$ & $=$ wing span, $\mathrm{ft}$. & $\alpha$ & $=$ angle-of-attack, rad or deg \\
\hline$C_{N}, C_{Y}$ & $=$ normal and side force coefficients & $\alpha_{0}$ & $=$ initial angle-of-attack in forced \\
\hline$C_{l}, C_{n}$ & $=$ rolling and yawing-moment coefficients & & oscillation experiments, rad or deg \\
\hline$C_{m}$ & $=$ pitching-moment coefficients & $\beta$ & $=$ sideslip angle, rad or deg \\
\hline & $=$ mean aerodynamic chord, $\mathrm{ft}$. & $\eta$ & $=$ state variable \\
\hline$F_{a_{\alpha}}, F_{a_{\beta}}$ & $=$ deficiency functions & $\sigma$ & $=$ standard error \\
\hline$f$ & $=$ frequency, $\mathrm{Hz}$ & $\tau$ & $=$ dummy integration variable \\
\hline$k$ & $=$ reduced $f, \pi b f / V$, or $\pi \bar{c} f / V$ & $\tau_{1}$ & $=$ non-dimensional time constant, $\frac{1}{h}\left(\frac{2 \mathrm{~V}}{h}\right.$ \\
\hline $\begin{array}{l}m \\
N\end{array}$ & $\begin{array}{l}=\text { no. of harmonics in Fourier expansion } \\
=\text { number of data points }\end{array}$ & $\omega$ & $=$ angular frequency, $\mathrm{rad} / \mathrm{sec}$ \\
\hline$p, q, r$ & $=$ roll, pitch, and yaw rates, $\mathrm{rad} / \mathrm{sec}$ & $\bar{C}_{a_{\gamma}}$ & $=$ in-phase coefficients, $\gamma=\alpha$ or $\beta$ \\
\hline $\begin{array}{l}R^{2} \\
S\end{array}$ & $\begin{array}{l}=\text { coefficient of determination } \\
=\text { reference } a r e a, \mathrm{ft}^{2}\end{array}$ & $\bar{C}_{a_{\zeta}}$ & $=$ out-of-phase coefficients, $\zeta=p, q$, or $r$ \\
\hline $\mathrm{T}$ & $=$ dimensional time constant, sec & $S S_{E}, S S_{r}$ & $=$ residual and total sum of squares \\
\hline Subscripts & & Superscri & ipts \\
\hline$A$ & $=$ amplitude & $\wedge$ & $=$ estimated value \\
\hline $\begin{array}{l}a \\
E\end{array}$ & $\begin{array}{l}=\text { aero forces and moments: } N, Y, l, m, \text { or } n \\
=\text { measured value }\end{array}$ & $\sim$ & $=$ mean value \\
\hline
\end{tabular}

\footnotetext{
${ }^{1}$ Senior Research Engineer, Dynamic Systems and Control Branch, MS 308, Associate Fellow.

${ }^{2}$ Senior Research Engineer, Configuration Aerodynamics Branch, MS 499, Associate Fellow.

${ }^{3}$ Research Aerospace Engineer, Configuration Aerodynamics Branch, MS 499.

${ }^{4}$ Senior Research Engineer, Flight Dynamic Branch, MS 308, Senior Member.

${ }^{5}$ Senior Research Engineer, Flight Dynamic Branch, MS 308, Senior Member.
} 


\section{Introduction}

$\mathrm{T}$ he National Aeronautics and Space Administration (NASA) has completed a number of programs, working with industry, academia, and other government agencies, to improve flight safety and in particular to reduce loss-ofcontrol accidents [1-5]. The NASA Technologies for Airplane State Awareness (TASA), a sub-project of the NASA System Wide Safety (SWS) Program, is continuing this research [6-7]. A number of studies have been completed considering a conventional mid-sized commercial transport with a low horizontal tail; however, the current efforts are focused on models appropriate for T-Tail, aft twin-engine, configurations. One aspect of this current work is to investigate high-fidelity aerodynamic models in stall regions of the flight envelope that can be used in simulation and stall training. In support of this goal, both analytical and experimental tools and techniques continue to be developed that facilitate efficient testing and estimation of high-fidelity mathematical models in the aerodynamically more complex stall regions.

Aerodynamic models are developed using aircraft System Identification (SID) and Computational Fluid Dynamics (CFD) tools. These tools and techniques are used to obtain aerodynamic models that allow prediction of nonlinear unsteady behaviors. SID tools are applied to both wind-tunnel experimental measurements and CFD simulated measurements of aircraft dynamic responses to obtain unsteady models suitable for flight dynamics simulations. Besides simulated dynamic responses, CFD provides insights into the flow physics that occur in unsteady regions of the flight envelope.

Efficient inputs for unsteady modeling have been well established for wind-tunnel forced-oscillation tests. Early studies [8] demonstrated the appropriate use of sinusoids, ramps, and wide-band inputs. Single-frequency sinusoidal inputs provide diagnostic information as well as facilitate estimation of unsteady models. Wide-band inputs provide a richer more efficient data source for estimation and ramp-and-hold inputs offer useful validation data. This discussion is also considered in later reports [9-10] that provide a broader discussion of unsteady modeling.

Since CFD can be computationally demanding, one hypothesis is that step inputs or ramps may be used to excite the indicial responses for SID modeling and produce less computationally demanding time histories compared to a series of single-frequency sinusoidal inputs at six or more different frequencies. This hypothesis reverses the standard wind-tunnel approach where the sinusoidal inputs are used for diagnostics and modeling and then ramps are used for validation data. To test this hypothesis, the same three types of inputs are applied in CFD simulation studies specifically for an unsteady modeling case identified for two generic aircraft configurations.

The two different generic aircraft configurations, shown in Figs. 1-2, are considered under dynamic test conditions. Fig. 1 shows a generic sub-scale fighter configuration called the Stability and Control Configuration (SACCON) [11] and Fig. 2 shows the NASA Generic T-Tail (GTT) configuration [6]. The SACCON aerodynamic model, obtained from wind tunnel measurements, is used to create a dynamic simulation with known parameters that allows alternative input designs to be directly evaluated. These results highlight possible best practices for estimating unsteady models from CFD simulated experiments. In support of the TASA study, CFD is used to expand on experimental results for the GTT water-tunnel tests. In this study, the focus is on the stalled region where unsteady behaviors are indicated in the longitudinal axis. Application of SID and CFD tools to the GTT tests demonstrate some of the issues, as well as advantages and disadvantages, these tools bring to aerodynamic modeling.

In this study two objectives are addressed. The first objective is to obtain a representative model for a NASA generic aircraft under stalled conditions with unsteady responses. For this objective, SID analysis is applied to CFD simulated response data using the GTT aircraft. The second objective is to evaluate the modeling techniques involved to provide some preliminary guidance for application of these methods to unsteady modeling using either experimental or analytical methods. Both SACCON and GTT model data are used to meet the second objective.

\section{Experimental Data}

A portion of the lateral dynamic data, collected for the SACCON model in the NASA Langley $14 \times 22$ Subsonic Tunnel in Hampton, Virginia, was selected for analysis in this study [11-12]. An internal six-component strain gauge balance was used for the force and moment measurements. The sample rate was $300 \mathrm{~Hz}$ with an anti-alias filter at 100 Hz. Additional digital filtering was performed with a low pass filter at $4 \mathrm{~Hz}$ for this study. The filter was run in both directions to ensure no phase error was added to the data. Frequency effects were explored in roll forced-oscillation for the configuration with a round leading edge and fixed transition (RLE-FT) at tunnel velocity of $60 \mathrm{fps}$. The selected roll-oscillatory data were obtained at four nominal oscillation angles of attack, $0^{\circ}, 14^{\circ}, 15^{\circ}$, and $20^{\circ}$, at ten different frequencies from $0.24 \mathrm{~Hz}$ to $1.0 \mathrm{~Hz}$, and with amplitude of $5^{\circ}$ in bank angle.

A portion of the longitudinal force-oscillation data [6] collected for the GTT model at the Boeing FlowVisualization Water Tunnel (FVWT) in Huntington Beach, CA, was used in this study. The tunnel velocity was set at $1 \mathrm{fps}$ and a sample rate was set at $40 \mathrm{~Hz}$. Appropriate analog filtering and a low-pass digital filter was applied at $3 \mathrm{~Hz}$. 
The digital filter was run in both directions to ensure no phase error was added to the data. All pitch forced-oscillations were at an amplitude of $5^{\circ}$ angle of attack. Although limited frequency effects were explored over a large range of angle of attack, from $0^{\circ}$ to $55^{\circ}$, an initial screening showed sensitivity to frequency to be a relative maximum at $16^{\circ}$ angle of attack. Based on this, the effects of eight different non-dimensional frequencies from 0.0079 to 0.045 were investigated at $16^{\circ}$ angle of attack, which is the focus for GTT modeling in this study.

\section{Computational Data}

CFD predictions were performed with the USM3D flow solver [13] that is part of the NASA Tetrahedral Unstructured Software System (TetrUSS) [14]. USM3D is a parallelized tetrahedral cell-centered, finite volume compressible Reynolds-averaged Navier-Stokes (RANS) flow solver. The term "cell centered" means that the finite volume flow solution is solved at the centroid of each tetrahedral cell. Inviscid flux quantities are computed across each tetrahedral cell face using various upwind schemes. Spatial discretization is accomplished by a novel reconstruction process, based on an analytical formulation for computing solution gradients within tetrahedral cells. The solution can be advanced in time by a second order "physical" time step scheme, a second order "dual" time step scheme, or to a steady-state condition by an implicit backward-Euler scheme. Several turbulence models are available including the one-equation Spalart-Allmaras (SA) model and several two-equation models. The two-equation models available are the Jones and Launder k- $\varepsilon$ model, Menter Shear Stress Transport (SST) model, nonlinear Algebraic Reynolds Stress Models (ARSM) of Girimaji and Shih/Zhu/Lumley, and the Wilcox 1988 k- $\omega$ model. Detached Eddy Simulation (DES) has been implemented in all of the turbulence models. A capability to trip the flow at specified locations on aerodynamic surfaces has been implemented for the k- $\varepsilon$ turbulence model, but fully turbulent flow was assumed for the results in this paper. USM3D has capabilities for overset grids and dynamic grid motion, the latter being used in the current study. Published guidelines [15] for computing dynamic forced oscillation solutions were employed for the CFD computations in this study. CFD simulations were generated that correspond to the pitch forcedoscillation wind tunnel tests performed on the GTT in the regions of interest.

\section{Aerodynamic Modeling}

Identifying an adequate aerodynamic model requires a clear definition of the requirements and intended use for that model, as well as an appropriately designed test to allow identification. A general model and recommended test techniques developed for nonlinear unsteady conditions has been developed over a number of years and is summarized in Ref. [9]. The following section presents some of those concepts that are indicated for the current study.

\section{A. General Unsteady Model}

Aerodynamic coefficients, $C_{N}, C_{m}, C_{Y}, C_{n}$, and $C_{l}$, are represented by $C_{a}$, where subscripts $a=N, m, Y, n$, or $l$. Model structures for the aerodynamic coefficients utilized in this study are given below. In this study, a linear unsteady model structure was used and found to be adequate for both longitudinal and lateral-directional axes. Under limited motion assumptions [9], this model structure allows estimated parameters to vary with states $\alpha$ and $\beta$, thus allowing a broad class of nonlinear aerodynamic responses to be captured with this model structure. Each lateraldirectional coefficient can be written as

$$
C_{a}(t)=C_{a}(0)+\int_{0}^{t} C_{a_{\beta}}(t-\tau) \dot{\beta}(\tau) d \tau+\frac{b}{2 V} \int_{0}^{t} C_{a_{p}}(t-\tau) \dot{p}(\tau) d \tau+\frac{b}{2 V} \int_{0}^{t} C_{a_{r}}(t-\tau) \dot{r}(\tau) d \tau
$$

and the longitudinal coefficients can be written as

$$
C_{a}(t)=C_{a}(0)+\int_{0}^{t} C_{a_{\alpha}}(t-\tau) \dot{\alpha}(\tau) d \tau+\frac{\bar{c}}{2 V} \int_{0}^{t} C_{a_{q}}(t-\tau) \dot{q}(\tau) d \tau
$$

In Eqs. (1a) and (1b) coefficients $C_{a_{\alpha}}(t), C_{a_{q}}(t), C_{a_{\beta}}(t), C_{a_{p}}(t)$, and $C_{a_{r}}(t)$ are the indicial functions and $C_{a}(0)$ is the initial value of $C_{a}$. Two assumptions [16] are adopted to simplify the model: (a) the effect of angular accelerations $\dot{q}, \dot{p}$, and $\dot{r}$ on any coefficient can be neglected and (b) the indicial functions in Eq. (1) can be expressed for the lateral-directional case as 


$$
C_{a_{\beta}}(t)=C_{a_{\beta}}(\infty)-F_{a_{\beta}}(t)
$$

and for the longitudinal case as

$$
C_{a_{\alpha}}(t)=C_{a_{\alpha}}(\infty)-F_{a_{\alpha}}(t)
$$

where $F_{a_{\alpha}}(t)$ and $F_{a_{\beta}}(t)$ are the deficiency functions and $C_{a_{\alpha}}(\infty)$ and $C_{a_{\beta}}(\infty)$ are the rates of change $C_{a}$ with $\alpha$ and $\beta$, respectively, evaluated in steady flow conditions.

The simplified model, which takes into account changes with respect to steady state, has the lateral-directional form

$$
C_{a}(t)=C_{a_{\beta}}(\infty) \beta(t)+\frac{b}{2 V} C_{a_{p}}(\infty) p(t)+\frac{b}{2 V} C_{a_{r}}(\infty) r(t)-\int_{0}^{t} F_{a_{\beta}}(t-\tau) \dot{\beta}(\tau) d \tau
$$

and the longitudinal form

$$
C_{a}(t)=C_{a_{\alpha}}(\infty) \alpha(t)+\frac{\bar{c}}{2 V} C_{a_{q}}(\infty) q(t)-\int_{0}^{t} F_{a_{\alpha}}(t-\tau) \dot{\alpha}(\tau) d \tau
$$

To obtain a model appropriate for identification and with a limited number of parameters, the deficiency function is assumed to be a simple exponential function

$$
F_{a_{\gamma}}=a e^{-b_{1} t}
$$

where $\gamma$ can be $\alpha$ or $\beta$ depending on axis being modeled. Models appropriate for an aircraft undergoing one degreeof-freedom forced oscillation in roll or yaw can be obtained using Eqs. (3) and (4). Considering one degree-of-freedom rolling motion in the tunnel

$$
C_{a}(t)=C_{a}[\phi(t), p(t)]
$$

where sideslip angle is related to the roll angle by the equation

$$
\beta(t)=\sin ^{-1}(\sin \alpha(t) \sin \phi(t))
$$

Combining Eqs. (3-6), the aerodynamic models can be formulated as

$$
C_{a}(t)=C_{a_{\beta}}(\infty) \beta(t)+\frac{b}{2 V} C_{a_{p}}(\infty) p(t)-a \int_{0}^{t} e^{-b_{1}(t-\tau)} \dot{\beta}(\tau) d \tau
$$

By introducing

$$
\eta(t)=\int_{0}^{t} e^{-b_{1}(t-\tau)} \dot{\beta}(\tau) d \tau
$$

and applying the Leibniz integral rule, the state space form of Eq. (7) can be written as

$$
\dot{\eta}(t)=-b_{1} \eta(t)+\dot{\beta}(t)
$$




$$
C_{a}(t)=C_{a_{\beta}}(\infty) \beta(t)+\frac{b}{2 V} C_{a_{p}}(\infty) p(t)-a \eta(t)
$$

From Eq. (7), a steady response can be written as

$$
C_{a}(t)=\bar{C}_{a_{\beta}} \phi_{A} \sin (\omega t)+\bar{C}_{a_{p}} \phi_{A} k \cos (\omega t)
$$

where $\phi_{A}$ is the amplitude of roll oscillation, $k$ is reduced frequency, and $\bar{C}_{a_{\beta}}$ and $\bar{C}_{a_{p}}$ are the in-phase and out-ofphase components, respectively. These components are related to the model parameters (aerodynamic derivatives) by the equations

$$
\begin{gathered}
\bar{C}_{a_{\beta}}=C_{a_{\beta}}(\infty) \sin \alpha_{0}-a \frac{\tau_{1}^{2} k^{2}}{1+\tau_{1}^{2} k^{2}} \sin \alpha_{0} \\
\bar{C}_{a_{p}}=C_{a_{p}}(\infty)-a \frac{\tau_{1}}{1+\tau_{1}^{2} k^{2}} \sin \alpha_{0}
\end{gathered}
$$

For one degree-of-freedom yaw oscillations in the tunnel

$$
C_{a}(t)=C_{a}[\psi(t), r(t)]
$$

where yaw and sideslip angles are related by the equation

$$
\beta(t)=\sin ^{-1}(-\cos \alpha(t) \sin \psi(t))
$$

The state space equations, Eqs. (16-17), can be written as

$$
\begin{gathered}
\dot{\eta}(t)=-b_{1} \eta(t)+\dot{\beta}(t) \\
C_{a}(t)=C_{a_{\beta}}(\infty) \beta(t)+\frac{b}{2 V} C_{a_{r}}(\infty) r(t)-a \eta(t)
\end{gathered}
$$

and the corresponding in-phase and out-of-phase components are expressed as

$$
\begin{gathered}
\bar{C}_{a_{\beta}}=C_{a_{\beta}}(\infty) \cos \alpha_{0}-a \frac{\tau_{1}^{2} k^{2}}{1+\tau_{1}^{2} k^{2}} \cos \alpha_{0} \\
\bar{C}_{a_{r}}=C_{a_{r}}(\infty)+a \frac{\tau_{1}}{1+\tau_{1}^{2} k^{2}} \cos \alpha_{0}
\end{gathered}
$$

For the longitudinal axis a similar derivation results in the following expressions. For one degree-of-freedom pitch oscillations in the tunnel

$$
C_{a}(t)=C_{a}[\theta(t), q(t)]
$$

where pitch and angle-of-attack are constrained by the physical test arrangement and thus related by the equality (for upright oscillations) 


$$
\alpha(t)=\theta(t)
$$

The state space equations can be written as

$$
\begin{gathered}
\dot{\eta}(t)=-b_{1} \eta(t)+\dot{\alpha}(t) \\
C_{a}(t)=C_{a_{\alpha}}(\infty) \alpha(t)+\frac{\bar{c}}{2 V} C_{a_{q}}(\infty) q(t)-a \eta(t)
\end{gathered}
$$

and the corresponding in-phase and out-of-phase components are expressed as

$$
\begin{gathered}
\bar{C}_{a_{\alpha}}=C_{a_{\alpha}}(\infty)-a \frac{\tau_{1}^{2} k^{2}}{1+\tau_{1}^{2} k^{2}} \\
\bar{C}_{a_{q}}=C_{a_{q}}(\infty)-a \frac{\tau_{1}}{1+\tau_{1}^{2} k^{2}}
\end{gathered}
$$

\section{B. Experiment Design Considerations}

Aircraft SID provides a large body of knowledge on best practices for identifying aerodynamic models from flight or ground-based experimental facilities. Aircraft SID methods, in general, are described in [17] and SID methods focused on estimating unsteady models in a ground-based environment are described in [9]. Practitioners know that a well-designed experiment is required to achieve useful and validated mathematical models. For example, forcedoscillation frequencies chosen based on short-period frequencies will provide useful damping data but will likely miss any rigid-body unsteady behaviors. As indicated by Eqs. $(19,25)$, as the frequencies become larger the unsteady term is diminished relative to the steady-flow damping term.

In general, careful input design with a wide range of amplitudes and frequencies will provide sufficient information content for complete model identification. Fig. 3 shows a flow chart of the SID process with large blue arrows added to highlight where the standard process is modified for unsteady modeling. At the bottom of the figure the concept of Harmonic Analysis is highlighted. This is a diagnostic tool, used in combination with single-frequency forcedoscillation tests, that allows detection of unsteady behaviors and identifies the angle of attack and frequency ranges where it may occur. In addition, this analysis provides some guidance on the degree of nonlinear behaviors that might be present. With this guidance, the appropriate model structures and parameter estimation methods can be selected. Also shown in Fig. 3 is a list of specialized inputs that work well for this type of modeling. Different parameter estimation methods may be applicable depending on how the unsteady behaviors manifest. A two-step regression is useful when unsteady behavior presents in both in-phase and out-of-phase components of the response, however, the aerodynamic model is assumed to be linear for that option. If the unsteady behavior is only present in the out-of-phase component, then nonlinear regression is utilized. Equation error and output error are generally applied to linear and nonlinear aerodynamic cases, respectively.

Fig. 4 shows examples of useful inputs for identification and validation of unsteady models. These types of inputs require a test facility that allows programmable motions. In general, sinusoidal inputs are suited for harmonic analysis, wide-band inputs for efficient modeling, and ramps for model validation data. A key characteristic of the two wideband inputs is the enforcement of a flat power spectrum. This ensures that enough excitation is provided evenly over the specified frequency band.

\section{Harmonic Analysis}

Harmonic analysis provides an excellent diagnostic tool. It allows detection of unsteady behaviors and defines the range of frequencies and angles of attack where unsteady behavior occurs. In addition, it gives some guidance on the degree of nonlinearities when present. A conventional aerodynamic model assumed in forced-oscillation testing is given by Eq. (11). The form used for harmonic analysis can be written as

$$
C_{a}(t)=A_{0}+\sum_{j=1}^{m} A_{j} \cos (j \omega t)+\sum_{j=1}^{m} B_{j} \sin (j \omega t)
$$


The unknown parameters, $A_{0}, A_{j}$, and $B_{j}$, are the Fourier series coefficients that are directly related to the in-phase and out-of-phase coefficients normally obtained in forced-oscillation experiments. Harmonic analysis provides estimates of these coefficients, their standard errors, and the coefficient of determination, $R^{2}$. Since the terms in this series are orthogonal, they do not change value when additional higher-order terms are added. Higher-order terms are necessary when the aerodynamic response is nonlinear. If the first-order harmonic case $(j=1)$ terms provide an adequate fit to the data then only a linear aerodynamic model is required. If, in addition, no frequency dependence is present, then only a conventional damping term is required in the model. This can be seen by considering Eqs. (10, 17, and 23), where three distinctly different terms model the static, steady-flow dynamic, and unsteady responses.

For the model with linear aerodynamics and $A_{0}=0$, the aerodynamic in-phase and out-of-phase components can be expressed in terms of the coefficients $A_{1}$ and $B_{1}$. For the roll oscillation case the expressions are

$$
\begin{gathered}
\bar{C}_{a_{\beta}}=\frac{B_{1}}{\phi_{A}} \\
\bar{C}_{a_{p}}=\frac{A_{1}}{k \phi_{A}}
\end{gathered}
$$

For the yaw oscillation case the expressions are

$$
\begin{gathered}
\bar{C}_{a_{\beta}}=\frac{B_{1}}{\psi_{A}} \\
\bar{C}_{a_{r}}=\frac{A_{1}}{k \psi_{A}}
\end{gathered}
$$

where $\beta$ is related to $\phi$ and $\psi$ by Eqs. (6) and (15). For pitch oscillation the expressions are

$$
\begin{gathered}
\bar{C}_{a_{\alpha}}=\frac{B_{1}}{\alpha_{A}} \\
\bar{C}_{a_{q}}=\frac{A_{1}}{k \alpha_{A}}
\end{gathered}
$$

Another key part of harmonic analysis that helps sort details about the required model structure is the use of the Coefficient of Determination, $R^{2}$. This statistical metric indicates the fraction of the variation in measured data explained by the model and is defined as

$$
R^{2}=1-S S_{E} / \mathrm{SS}_{r} \quad 0<R^{2}<1
$$

where

$$
S S_{E}=\sum_{i=1}^{N}\left[C_{a_{E}}(i)-\hat{C}_{a}(i)\right]^{2}
$$

is the residual sum of squares and

$$
S S_{r}=\sum_{i=1}^{N}\left[C_{a_{E}}(i)-\tilde{C}_{a}(i)\right]^{2}
$$


is the total sum of squares. $C_{a_{E}}(i), \hat{C}_{a}(i)$, and $\tilde{C}_{a}(i)$ are the measured, estimated, and mean values, respectively.

\section{Parameter Estimation Method}

Parameter estimation for this study used a variation on an output-error method [9] in the time domain. Output error in the time domain is a more general method that allows estimation of a full unsteady model that may have linear or nonlinear terms in the model. In this study, the model equations are given by the state-space equations, noted in section V.A, corresponding to the axis of interest. Eqs. (9) and (10) were applied to lateral axis examples and Eqs. (22) and (23) were applied to the longitudinal axis examples. Measured time histories of the inputs, $\alpha$ or $\beta$, and outputs, $C a$, for each of the frequencies tested are combined or "stacked" to ensure all the frequency content is included in the time domain estimation process. Equation integrations must be performed separately for each oscillation test but a single cost function is used to capture the overall model effectiveness. Application of the general output-error method for aircraft is explained and relevant software is provided in Ref. [18].

\section{Results and Discussion}

The purpose of this analysis is to assess the various input signals in terms of the resulting parameter estimation quality and computational efficiency. Model identification is performed using the SACCON model, based on wind tunnel data [11, 12], and for the GTT model using both CFD-simulated data and water-tunnel data [6].

However, before using CFD-simulated data, a separate simulated experiment was performed to assess the identifiability of using SID methods applied to ramp and hold experiments versus sinusoidal or wide-band experiments. To accomplish this first step, a simulation of unsteady responses for SACCON is developed to allow estimation of known model parameters. These simulated data are obtained using Eqs. (9-10) with white noise added to allow a more realistic modeling problem. The noise level is relatively low and produces a signal-to-noise ratio of approximately 50:1. The quality of the estimated model reflects the quality of the various inputs. Results of this simulated study are provided in section VI.B. After the SACCON study, analysis of the best approach for input design and unsteady model estimation is extended using CFD-simulated data for GTT by applying the same three types of inputs. The latter case considers more complex aerodynamic behaviors.

\section{A. Harmonic Analysis}

The presence of unsteady behavior and the angles of attack where this aerodynamic behavior exists are indicated when the values of the in-phase and out-of-phase components show frequency dependence. If corresponding values of $R^{2}$ for the first-order harmonics are sufficiently high (practical experience suggests that values of $R^{2} \geq 0.8$ are usually sufficient in a wind-tunnel environment) then a linear model structure is indicated. However, if $R^{2}$ values are low, then further investigation is necessary to determine whether higher harmonics are required due to nonlinearities or simply the presence of relatively high noise. Estimates of $R^{2}$ are influenced by the value and number of Fourier coefficients (harmonic order) in Eq. (26) and the measurement noise, captured in Eq. (34). Because the harmonic sinusoids are mutually orthogonal, the estimates of $A_{j}$ and $B_{j}$ will not change with the number of model terms included. Changes will only appear in the corresponding standard errors and residuals defined by Eq. (34). This makes $R^{2}$ an effective diagnostic tool to discern the adequacy of a linear first-order model against nonlinear higher harmonic models.

\section{Harmonic Analysis for SACCON model, Wind-Tunnel Test}

Harmonic analysis was performed on $5^{\circ}$ amplitude roll oscillatory wind-tunnel data for the SACCON model. Harmonic analysis for this case is presented in Fig. 5. This analysis was originally presented in [12] and is provided again to support this study. Fig. 5 shows both out-of-phase and in-phase components of the roll moment and $R^{2}$ against angle of attack at different frequencies. The results show little or no frequency dependence for angles of attack at $15^{\circ}$ and below and therefore no unsteady aerodynamic effect. Frequency dependence is indicated for an angle of attack of $20^{\circ}$, so an unsteady model is required at this angle of attack. $R^{2}$ values at $\alpha=20^{\circ}$ indicate that a linear aerodynamic model is sufficient for this case. This representative unsteady model will be the basis for a simulated test of effectiveness for the three main types of inputs.

\section{Harmonic Analysis for GTT model, Water-Tunnel Test}

Harmonic analysis was applied to measured data from the GTT model in $5^{\circ}$ amplitude, pitch forced-oscillation, water-tunnel testing. Limited frequency sets were used to sweep a large angle of attack range. Investigators determined that $16^{\circ}$ angle of attack was a key location for frequency dependent behavior. Since the purpose of this paper is focused 
on modeling unsteady conditions, only $16^{\circ}$ angle of attack is considered. Table 1 presents the forced-oscillation frequencies considered and the corresponding non-dimensional frequencies.

Table 1. Forced-oscillation frequencies for GTT study in Boeing FVWT.

\begin{tabular}{|c|l|l|l|l|l|l|l|l|}
\hline & 1 & 2 & 3 & 4 & 5 & 6 & 7 & 8 \\
\hline$f(\mathrm{~Hz})$ & 0.0117 & 0.0178 & 0.0234 & 0.0296 & 0.0370 & 0.0468 & 0.0593 & 0.0667 \\
\hline$k$, non-dimensional & 0.0079 & 0.0120 & 0.0158 & 0.0200 & 0.0250 & 0.0316 & 0.0400 & 0.0450 \\
\hline
\end{tabular}

Harmonic analysis results for this case are shown in Figs. 6a and 6b. Figure 6a presents the analysis for normal force coefficient, $C_{N}$, and Fig. $6 \mathrm{~b}$ presents the analysis for pitching moment coefficient, $C_{m}$. Frequency dependence is verified at $16^{\circ}$ angle of attack and since all $R^{2}$ values are above $80 \%$ a linear unsteady model is suggested. For the low frequency case, where the poorest signal/noise is expected, the $R^{2}$ values are still very good for a first-order harmonic model, confirming that a linear unsteady model should be adequate at this angle of attack. Table 2 presents $R^{2}$ values for a first-order harmonic model at each frequency.

Table 2. First-order harmonic model adequacy based on $R^{2}$.

\begin{tabular}{|l|l|l|l|l|l|l|l|l|}
\hline & 1 & 2 & 3 & 4 & 5 & 6 & 7 & 8 \\
\hline$k$ & 0.0079 & 0.0120 & 0.0158 & 0.0200 & 0.0250 & 0.0316 & 0.0400 & 0.0450 \\
\hline$R^{2}$ for $C N$ & 84.7 & 93.4 & 95.0 & 96.7 & 98.1 & 97.9 & 98.9 & 98.9 \\
\hline$R^{2}$ for $C m$ & 80.9 & 90.3 & 90.2 & 89.8 & 91.9 & 95.2 & 97.4 & 98.5 \\
\hline
\end{tabular}

Figures 7-8 present "phase plots" with first-order harmonic models for $C_{N}$ and $C_{m}$ at low and high frequencies. The first-order harmonic model is shown as a regular ellipse (red line) against the measured data (blue "+" signs). The model fits the response data reasonably well especially for the higher frequencies. The lower frequency case demonstrates the higher noise levels, presence of other harmonics, and some nonlinearity. Other harmonics are often present in forced-oscillation testing due to a variety of sources, but a common source is structural vibration. In the final modeling analysis, higher harmonics and nonlinearities are considered only if required. In this case, $R^{2}$ values are very good and imply that a first-order linear unsteady model is adequate. Investigators found that attempting frequencies lower than shown produced questionable results with poor signal-noise conditions. Consequently those data were eliminated.

\section{Harmonic Analysis for GTT model, CFD Simulated Test}

Harmonic analysis was applied to dynamic data from the GTT model in $5^{\circ}$ amplitude, pitch forced-oscillation, CFD simulated testing. Forced oscillations of the same frequencies, appropriately scaled by $k$, were continued sufficiently long to ensure steady harmonic motions were achieved. Based on wind and water-tunnel results, investigators focused on $16^{\circ}$ angle of attack as a key location for less benign frequency dependent behavior. $R^{2}$ from harmonic analysis of the same 8 frequencies is shown in Table 3 . The table reveals that normal force is likely well modeled using a linear unsteady model. However, the pitching moment case is notably different.

Table 3. First-order harmonic model adequacy based on $R^{2}$.

\begin{tabular}{|l|l|l|l|l|l|l|l|l|}
\hline & 1 & 2 & 3 & 4 & 5 & 6 & 7 & 8 \\
\hline$k$ & 0.0079 & 0.0120 & 0.0158 & 0.0200 & 0.0250 & 0.0316 & 0.0400 & 0.0450 \\
\hline$R^{2}$ for $C N$ & 88.5 & 86.0 & 93.7 & 94.4 & 94.8 & 97.5 & 98.4 & 98.6 \\
\hline$R^{2}$ for $C m$ & 20.2 & 28.3 & 35.3 & 44.6 & 55.9 & 64.6 & 77.2 & 83.4 \\
\hline
\end{tabular}

Table 4 shows the third-order harmonic models the responses reasonably well based on $R^{2}$ values. The need for higher harmonics indicates that some nonlinearities or very poor signal-to-noise may be present. The significantly better fit for third-order harmonics also implies nonlinearities may be cubic in nature.

Table 4. Third-order harmonic model adequacy based on $R^{2}$.

\begin{tabular}{|l|l|l|l|l|l|l|l|l|}
\hline & 1 & 2 & 3 & 4 & 5 & 6 & 7 & 8 \\
\hline$k$ & 0.0079 & 0.0120 & 0.0158 & 0.0200 & 0.0250 & 0.0316 & 0.0400 & 0.0450 \\
\hline$R^{2}$ for $C N$ & 97.3 & 94.5 & 98.6 & 99.5 & 99.1 & 99.3 & 99.7 & 99.8 \\
\hline$R^{2}$ for $C m$ & 83.4 & 86.5 & 90.4 & 97.5 & 96.8 & 97.1 & 98.6 & 98.9 \\
\hline
\end{tabular}


Figures 9-10 show the "phase plots" for both first and third-order harmonic analysis. In these plots the CFD-simulated measurements are indicated by the blue "+" symbols, green shows the first-order harmonic model and red shows the third-order model. The change in sample rates for the measured data is associated with the very high sample rates produced in a CFD analysis. In order to produce very fine time-accurate responses, CFD analysis effectively results in sample rates with order of magnitude of several thousand Hertz. In CFD simulations, accuracy is also achieved by specifying the samples per cycle of oscillation. This in turn produces sample rates that vary according the frequency of oscillation. For flight dynamics analysis of forced-oscillation testing, appropriate sample rates for rigid-body, unsteady responses are typically in the range of 50 to $200 \mathrm{~Hz}$. Consequently all the CFD response data has been decimated to produce effective sample rates less than $250 \mathrm{~Hz}$. In addition, the plots of CFD responses in Figs. 9-10 have also been further reduced by skipping points and by only showing the mean response to avoid blotting out the response pattern.

A key result shown in Figs. 9-10 is the presence of nonlinearities and higher-order responses beyond the thirdharmonic model. These dynamics are not of interest in this study where only a representative model that captures the primary responses is desired. In general, if the parameters in any of the three terms in output Eq. (23) require substantially more than a cubic model, then it is likely that either a different model structure is required, such as demonstrated in Ref. [19], or possibly a smaller amplitude oscillation should be considered to allow more adequate modeling in that region of the flight envelope. High-order polynomials require more parameters to be estimated, leading to higher demands on the information content of the data and in turn, information content depends heavily on the inputs chosen. The following sections will consider three inputs to demonstrate their utility.

\section{B. Unsteady Model Identification}

Three modeling problems are considered in this section: (1) using simulated SACCON data, produced with known model parameters that allows direct evaluation of the three input forms, (2) using GTT water tunnel data to estimate an unsteady model for the T-Tail configuration, and (3) using CFD simulated tests of the GTT to obtain unsteady models.

\section{Model Identification for SACCON, Simulation Test}

Model estimation using wind tunnel data was performed in a previous study [12] for the SACCON model. Table 5 presents the model parameters estimated from that previous study. Those model parameters are used again in this study to create a dynamic simulation based on the model structure presented in Eqs. (9-10). Parameters values were rounded, shown in Table 6, to allow comparisons against well-defined, known parameter values.

Sinusoidal, ramp-and-hold, and Schroeder inputs were applied in this simulation. For sinusoidal forced oscillations the amplitude was set at $5^{\circ}$ in roll and eight forced-oscillation frequencies were set at 0.24, 0.36, 0.44, 0.55, 0.66, 0.70, 0.85 , and $1.0 \mathrm{~Hz}$. For ramp-and-hold simulations an amplitude of $5^{\circ}$ was used along with 4 different ramp rates of 1 , 10,20 and 30 degrees/second. A Schroeder sweep was designed to both span the frequency set and provide sufficient frequency resolution.

Table 5. Linear Unsteady Aerodynamic Model for SACCON at $\alpha_{0}=20 \mathrm{deg}$.

\begin{tabular}{|l|l|l|l|l|l|}
\hline Parameter & $C_{l_{\beta}}$ & $C_{l_{p}}$ & $a$ & $b_{1}$ & $\tau_{1}$ \\
\hline Estimate, $\hat{\theta}$ & 0.57 & -0.40 & 0.75 & 3.68 & 6.37 \\
\hline Standard dev, $\hat{\sigma}$ & 0.05 & 0.047 & 0.04 & 0.34 & 0.58 \\
\hline
\end{tabular}

Table 6. Linear Unsteady Aerodynamic Model for SACCON Simulation at $\alpha_{0}=20 \mathrm{deg}$.

\begin{tabular}{|l|l|l|l|l|l|}
\hline Parameter & $C_{l_{\beta}}$ & $C_{l_{p}}$ & $a$ & $b_{1}$ & $\tau_{1}$ \\
\hline Simulated $\theta$ & 0.60 & -0.40 & 0.70 & 4.0 & 5.954 \\
\hline
\end{tabular}

To simulate the wind tunnel environment white noise was added to the responses. Root-mean-square (RMS) signal-to-noise ratio was approximately 50:1. This provided a relatively benign test environment for the estimation tools since in an actual test some degree of other harmonics and nonlinearities would normally be present. However, under these benign circumstances any differences in performance of the estimation process will highlight the relative quality of the three input types. As an example, Fig. 11 shows an input and response for the case where the fastest ramp-and-hold input is applied. The input with the highest ramp rate of 30 degrees/second closely approximates a step change in roll. The roll moment response is shown on the right side, the forced input is shown on the lower left-hand side, and the deficiency function, $\eta(t)$, is shown on the upper left side. The lower left graphic presents both the forced 
positon of the model, a change in roll angle, $\phi(t)$, as well as the change in sideslip, $\beta(t)$, which is the driving aerodynamic input function.

Table 7a shows the estimated parameters for the simulation model ("true" values) and the three estimated models from simulated data using three different inputs. For the sinusoidal input test, 6 cycles of data for each of the 8 frequencies were stacked and used for analysis and for the ramp test 4 different ramp rates were stacked for analysis. The Schroeder sweep was a 25-second, single-input signal, providing a flat power spectrum and very high frequency resolution. All three inputs provide adequate models based on $R^{2}$ values, however, the Schroeder sweep and sinusoidal inputs supported estimation of parameters much closer to the true values than the ramp-and-hold inputs. Table $7 \mathrm{~b}$ shows the percent error for each case and highlights the superior nature of either wide-band or multiple sinusoidal inputs.

Table 7a. Estimated Linear Unsteady Aerodynamic Models from SACCON Simulation at $\alpha_{0}=20 \mathrm{deg}$.

\begin{tabular}{|c|l|l|l|l|l|l|l|}
\hline Parameter & Input type & $C_{l_{\beta}}$ & $C_{l_{p}}$ & $a$ & $b_{1}$ & $\tau_{1}$ & $R^{2}$ \\
\hline Simulated $\theta_{\text {true }}$ & -- & 0.60 & -0.40 & 0.70 & 4.0 & 5.954 & 1.0 \\
\hline Estimate, $\hat{\theta}$ & sinusoids & 0.595 & -0.408 & 0.688 & 3.976 & 5.982 & 0.99 \\
$(\hat{\sigma})$ & & $(0.0002)$ & $(0.0006)$ & $(0.0006)$ & $(0.0041)$ & $(0.007)$ & \\
\hline $\begin{array}{c}\text { Estimate, } \hat{\theta} \\
(\hat{\sigma})\end{array}$ & Ramp- & 0.598 & -0.321 & 0.784 & 4.471 & 5.319 & 0.99 \\
\hline and-hold & $(0.0001)$ & $(0.0023)$ & $(0.0034)$ & $(0.020)$ & $(0.024)$ & \\
$(\hat{\sigma})$ & Schroeder & 0.594 & -0.404 & 0.692 & 4.010 & 5.930 & 0.99 \\
\hline
\end{tabular}

Table 7b. Percent Error for Models Terms from SACCON Simulation at $\alpha_{0}=20 \mathrm{deg}$.

\begin{tabular}{|c|l|l|l|l|l|l|}
\hline Parameter & Input type & $C_{l_{\beta}}$ & $C_{l_{p}}$ & $a$ & $b_{1}$ & $\tau_{1}$ \\
\hline $\begin{array}{c}\% \text { Error, } \\
\left(\widehat{\theta}-\theta_{\text {true }}\right) / \theta_{\text {true }}\end{array}$ & sinusoids & $-0.83 \%$ & $2.0 \%$ & $-1.71 \%$ & $-0.60 \%$ & $0.47 \%$ \\
\hline $\begin{array}{c}\% \text { Error, } \\
\left(\hat{\theta}-\theta_{\text {true }}\right) / \theta_{\text {true }}\end{array}$ & $\begin{array}{l}\text { Ramp- } \\
\text { and-hold }\end{array}$ & $-0.33 \%$ & $-19.75 \%$ & $12.0 \%$ & $11.78 \%$ & $-10.67 \%$ \\
\hline $\begin{array}{c}\% \text { Error, } \\
\left(\hat{\theta}-\theta_{\text {true }}\right) / \theta_{\text {true }}\end{array}$ & $\begin{array}{l}\text { Schroeder } \\
\text { sweep }\end{array}$ & $-1.0 \%$ & $1.0 \%$ & $-1.14 \%$ & $0.25 \%$ & $-0.40 \%$ \\
\hline
\end{tabular}

\section{Model Identification for GTT Model, Water-Tunnel Test}

Water tunnel tests provided flow visualization and dynamic data. Although the focus of this study is primarily on using CFD response data, results of the unsteady model analysis for GTT from the water tunnel are also provided. As a sample result, Table 8 shows the model parameters estimated for $C_{m}$ at $16^{\circ}$ angle of attack. Trim settings and biases are removed from the data before analysis. Consequently, estimates of $C_{m 0}$ are effectively zero.

Table 8. Linear Unsteady Aerodynamic Model for GTT at $\alpha_{0}=16 \mathrm{deg}$.

\begin{tabular}{|c|l|l|l|l|l|l|l|}
\hline Parameter & $C_{m 0}$ & $C_{m \alpha}$ & $C_{m q}$ & $a$ & $b_{1}$ & $\tau_{1}$ & $R^{2}$ \\
\hline Estimate, $\hat{\theta}$ & 0.0 & 0.640 & -63.8 & -1.66 & 0.467 & 19.95 & 0.94 \\
\hline Standard dev, $\hat{\sigma}$ & 0.0001 & 0.0029 & 0.38 & 0.039 & 0.0084 & 0.36 & --- \\
\hline
\end{tabular}

Figures 12-13 provide the linear unsteady model prediction against measured responses for a low and high frequency, respectively. Both predicted and measured responses are shown as changes from the nominal ("trim") oscillation condition at $\alpha_{0}=16$ degrees. Nominal conditions are subtracted from the responses. Included in the figures are blue dashed lines, representing the linear estimated static model. The model provides an adequate representation of the dynamics measured in the water tunnel.

\section{Model Identification for GTT Model, CFD Simulated Test}

The CFD solutions were computed using USM3D with Spalart-Allmaras turbulence model on a half-span GTT grid of 28.8 million tetrahedral cells. Since USM3D is formulated as a compressible Reynolds-Averaged Navier- 
Stokes flow solver, numerical convergence and accuracy degrades rapidly for very low Mach numbers. Since the $12 \mathrm{FT}$ wind tunnel data was measured at $M_{\infty}=0.052$, a higher $M_{\infty}=0.126$ was prescribed for the USM3D solutions to insure good numerical convergence. Furthermore, the CFD geometry is based on the larger subscale flying model with $\bar{c}=21.204$ inches whereas the wind-tunnel (WT) model has a $\bar{c}=7.7612$ inches. Hence, it was necessary to scale the CFD motion time scale to match the reduced frequencies of the WT data. Since reduced frequency is simply the ratio of a velocity at the end of a rotating reference length relative to the oncoming velocity, the scaled CFD frequency is defined in Eq. (36). Once a solution is complete, then the time is rescaled back to the wind-tunnel conditions.

$$
f_{C F D}=f_{W T}\left(\bar{c} / M_{\infty}\right)_{W T}\left(M_{\infty} / \bar{c}\right)_{C F D}
$$

The pitch forced oscillation (FO) cases were run for two full cycles with 1800-time steps per cycle, for a total of 3600-time steps. Each time step was converged with 40 subiterations. The ramp-and-hold solutions were run with a characteristic time step, $\Delta \mathrm{t}^{*}=\Delta \mathrm{t} \bullet \mathrm{M}_{\infty} / \bar{c}=0.01$ using 20 subiterations until the final $C_{N}$ and $C_{m}$ transients settled out. The final number of time steps is noted in Table $9 \mathrm{c}$. Schroeder sweeps were eliminated from this analysis since CPU time to create a desirable 50-second sweep with adequate frequency resolution created an unacceptable delay. Processing was estimated to be on the order of weeks. Additional study is required to determine methods to speed up CFD calculations as well as checking estimation accuracy using sweeps of shorter duration.

The objectives for using CFD simulated data were to obtain unsteady models to help corroborate or gain insights into the experimental results and to evaluate test and modeling techniques. Besides accuracy, one aspect of technique evaluation is to consider the efficiency or resources required to obtain data. Two metrics to consider are the Central Processing Unit (CPU) usage and the wall-clock time of the computations. These metrics varied according to the type of input chosen, since simulations were run until sinusoids reached steady-harmonic oscillations and ramps ran until transient responses settled. All cases are for the nominal $16^{\circ}$ angle of attack. CFD calculations are all performed on the NASA Langley Research Center K3 machine using 144 processors. Table 9a shows the oscillation frequencies chosen for the analysis. Tables $9 \mathrm{~b}$ and $9 \mathrm{c}$ show the CPU time required for each input type.

Table 9a. Non-dimensional Forced-Oscillation Frequencies for 2-Cycle Sinusoids.

\begin{tabular}{|l|l|l|l|l|l|l|}
\hline Input type & Sinusoid, & Sinusoid, & Sinusoid, & Sinusoid, & Sinusoid, & Sinusoid, \\
& $\mathrm{k}=0.0079$ & $\mathrm{k}=0.012$ & $\mathrm{k}=0.0158$ & $\mathrm{k}=0.025$ & $\mathrm{k}=0.0316$ & $\mathrm{k}=0.040$ \\
\hline
\end{tabular}

Table 9b. CFD Computational Requirements for GTT 2-cycle Sinusoids, $\alpha_{0}=16^{\circ}, \alpha_{A}=5^{\circ}, 28.8 \mathrm{M}$ cells .

\begin{tabular}{|l|c|c|}
\hline & $\begin{array}{c}\text { K3 CPU time } \\
\text { (hours) }\end{array}$ & K3 Wall-clock time, 144 processors \\
\hline Initial Static & 1104 & 7.7 hours \\
\hline Single-frequency, 3600 time steps & 8350 & 2.4 days \\
\hline Six-frequency & 50100 & $\sim 1$ week (cases run in parallel) \\
\hline
\end{tabular}

Table 9c. CFD Computational Requirements for GTT Ramp-and-Holds, $\alpha_{0}=11^{\circ}, \alpha_{A}=5^{\circ}, 28.8 \mathrm{M}$ cells.

\begin{tabular}{|l|c|c|c|c|}
\hline & No. time steps & $\Delta \mathrm{t}^{*}$ & $\begin{array}{c}\text { K3 CPU time } \\
\text { (hours) }\end{array}$ & K3 Wall-clock time, 144 processors \\
\hline Initial Static & 6000 & 0.1 & 2200 & 15.3 hours \\
\hline Ramp 30 d/s & 6520 & 0.01 & 6454 & 1.9 days \\
\hline Ramp 20 d/s & 7275 & 0.01 & 7180 & 2.1 days \\
\hline Ramp 10 d/s & 9517 & 0.01 & 6662 & 1.9 days \\
\hline Ramp 1 d/s & 5053 & 0.1 & 6530 & 1.9 days \\
\hline
\end{tabular}

Based on harmonic analysis results, shown in Tables 3-4, the more challenging modeling problem is presented in the pitching moment data. In addition, Figs. 9-10 presented a clear indication that $C_{m}$ will likely require a nonlinearunsteady model. The lowest frequency case, in Fig. 9, also demonstrated that some of the responses included higherorder dynamics not of interest to this study. To make this paper more tractable, the remaining discussion will focus on modeling the $C_{m}$ response.

Results of the unsteady model analysis for GTT are given in Table 10. Trim settings are removed from the data so $C_{m o}$ reflects a residual bias term not the nominal aerodynamic moment. CFD forced-oscillation data was restricted to 6 frequencies to represent a typical minimum set of data available for modeling and to allow additional cases for validation tests. Table 10a provides the estimated static model for each input type. Table 10b provides the 
corresponding unsteady model. In order to get an adequate model, the static model required a cubic polynomial, as expected based on harmonic analysis of the sinusoidal responses. The ramp inputs forced motion over half the range of angle of attack, as compared to the sinusoids, to ensure completion at $\alpha=16^{\circ}$. Consequently, the data cover a slightly different range and required different model for the static and steady-flow damping terms.

Table 10a. Estimated Static Model from CFD Simulation of GTT at $\alpha_{0}=16 \mathrm{deg}$.

\begin{tabular}{|c|c|c|c|c|c|}
\hline Parameter & Input type & $C_{m_{0}}$ & $C_{m_{\alpha}}$ & $C_{m}{ }_{\alpha^{2}}$ & $C_{m_{\alpha}}$ \\
\hline $\begin{array}{c}\text { Estimate, } \hat{\theta} \\
(\hat{\sigma})\end{array}$ & sinusoids & $\begin{array}{l}0.0313 \\
(0.0006)\end{array}$ & $\begin{array}{l}1.1580 \\
(0.0314)\end{array}$ & $\begin{array}{l}-8.1984 \\
(0.1280)\end{array}$ & $\begin{array}{l}-146.5776 \\
(2.9366)\end{array}$ \\
\hline $\begin{array}{c}\text { Estimate, } \hat{\theta} \\
(\hat{\sigma})\end{array}$ & $\begin{array}{l}\text { Ramp- } \\
\text { and-hold }\end{array}$ & $\begin{array}{l}0.0253 \\
(0.0019)\end{array}$ & $\begin{array}{l}2.9802 \\
(0.0810)\end{array}$ & $\begin{array}{l}33.5548 \\
(1.8051)\end{array}$ & $\mathrm{n} / \mathrm{a}$ \\
\hline
\end{tabular}

Table 10b. Estimated Unsteady Model from CFD Simulation of GTT at $\alpha_{0}=16 \mathrm{deg}$.

\begin{tabular}{|c|l|l|l|l|l|l|l|}
\hline Parameter & Input type & $C_{m_{q}}$ & $C_{m_{q \alpha}}$ & $a$ & $b_{1}$ & $\tau_{1}$ & $R^{2}$ \\
\hline $\begin{array}{c}\text { Estimate, } \hat{\theta} \\
(\hat{\sigma})\end{array}$ & sinusoids & -25.4114 & 421.4108 & 0.3517 & 1.3186 & 138.5132 & 0.76 \\
\hline $\begin{array}{c}\text { Estimate, } \hat{\theta} \\
(\hat{\sigma})\end{array}$ & Ramp- & $(0.4104)$ & $(6.7848)$ & $(0.0211)$ & $(0.1487)$ & $(15.6203)$ & \\
\hline
\end{tabular}

Figures 14-15 show how significantly the predicted response changes from a classic linear aerodynamic model when model parameters are expanded to higher-order polynomial terms. The figures provide the nonlinear-unsteady model prediction against CFD simulated "measured" forced-oscillation responses at a low and high frequency, respectively. Both predicted (red line) and measured responses (green "+") are shown as changes from the nominal oscillation condition at $\alpha_{0}=16$ degrees. Included in the figures are blue lines, representing the estimated static model. The model provides an adequate representation of the dynamics provided by CFD simulation given the objective of obtaining a representative model.

A validation test of the unsteady model from sinusoidal data is shown in Fig. 16. In this case, the final model is used to predict responses for the frequency case $(k=0.020)$ not used for estimation. The results show the model is adequate as a representative model that captures the dominate features of the response indicated by CFD simulation. The validation test produced an $R^{2}$ value of $79.7 \%$.

Identifying a model based on ramp data presented several difficulties. Figure 17 helps explain the issues for this type of data. The original CFD data in these graphics have been decimated so that the sample rates are well below 250 Hz. In each graphic, significant nonlinear and higher-order responses are observed. The data point that appears to be an outlier for the 30 degree/second case, is reflecting the actual response behavior as the ramp changes to a constant angle of attack. With higher sample rate data, the step-like change and spike in $C_{m}(t)$ is revealed as part of the actual very high-frequency response. However, for model identification, all CFD data was further filtered to further reduce or eliminate these very high-order effects. The ramp data with a 1 degree/second rate was eliminated since it contained insufficient unsteady content to allow estimation of the model. This was likely due to the nonlinear high-order effects dominating the response. An example of the final model based on the remaining three ramps is presented in Fig. 18, showing the measured and predicted responses for the 30 degrees/second case.

\section{Concluding Remarks}

In this study an initial set of results was obtained to make an assessment of the tools and techniques for unsteady model estimation and to obtain a representative model for the NASA GTT aircraft at $16^{\circ}$ angle of attack. The focus was on using three different, commonly used, inputs for experimental testing: (1) sinusoidal forced-oscillation, (2) ramp-and-hold, and (3) Schroeder sweep. The three inputs were applied to the SACCON model in a simulated dynamic test to provide an initial screening of the test methods. To ensure the tests were sufficiently challenging, a point in the GTT flight envelope was chosen with distinctly nonlinear and unsteady behaviors. The first two input types were successfully applied to the NASA GTT model in CFD simulation. The third input was eliminated due to an unacceptable computational time. Future work is underway to identify a better solution strategy for wide-band inputs that reduces the computational expense. 
The results of this initial study lead to the conclusion that the sinusoidal forced-oscillation method is the preferred method. First, it provides a convenient diagnostic tool. Second, it performs well by ensuring frequency content is obtained with sufficient energy evenly over the range of interest. As a practical matter, the application of singlefrequency sinusoids to a dynamic system has the advantage of revealing nonlinearities as a result of the characteristics of linear systems. For a linear system, a single-frequency input results in a single-frequency output, although the response may lag and be attenuated. In contrast, nonlinear systems can respond to a single frequency input with multiple harmonics. The wide-band input (Schroeder sweep) also appears to be very effective; however, further testing is required for CFD applications to confirm its full utility. Adjusting grid size, number of processors, and the wideband input design itself can change the conclusions from this initial study.

In theory, indicial models are best obtained through a step input; however, as a practical matter involving real testing environments, both analytical and experimental, the results of this study indicate that very little measurement noise, higher-order dynamics, or nonlinearities can substantially limit a step or ramp input approach. From a frequency domain perspective this make sense. Although a step input model (e.g., Fourier series) requires many frequencies to match the step, it does not spread the energy evenly over the frequencies, unlike the wide-band inputs described in this study. However, no attempt was made to optimize the ramp inputs, so pure steps may provide better identification in a CFD environment, especially in regions where nonlinear behaviors are limited. As shown in the simulated SACCON data, a selection of ramp rates does not provide inputs as effective as either sinusoidal or wide-band sweeps. From the results in this study, ramps are not reliable inputs in very nonlinear, high-order, response portions of the flight envelope.

In any modeling problem, a balance between over fitting and under fitting is always present. The over-fitting problem is often addressed with a number of statistical tools and, as a practical matter, model prediction is typically improved with a more parsimonious model. Unique to the study, is the objective to find representative models for the GTT class of vehicle, not specific, high-fidelity, models for a given experimental or analytical test. In this case, a subject matter expert or simulation engineer using the models would provide guidance as to the level of complexity that makes sense for their application. For the current model structure, going beyond cubic expansions for each parameter may lead to a requirement for more data to sufficiently characterize that more complex model. If that level of complexity is required, a complete change in the assumed model structure may be more efficient.

\section{Acknowledgments}

The authors extend their appreciation to the Vehicle Safety SE209 element of the Technologies for Airplane State Awareness project, a sub-project in the NASA System Wide Safety Program.

\section{References}

[1] Cunningham, Kevin, Foster, John V, Shah, Gautam H., and Stewart, Eric C., "Simulation Study of a Commercial Transport Airplane During Stall and Post-Stall Flight,” SAE Technical Paper Series 2004-01-3100, November 2004.

[2] Foster, John V., Cunningham, Kevin, Fremaux, Charles M., Shah, Gautam H., and Stewart, Eric C., "Dynamics Modeling and Simulation of Large Transport Airplanes in Upset Conditions," AIAA Guidance Navigation, and Control Conference, AIAA 2005-5933, August 2005.

[3] Belcastro, Christine M. and Foster, John V., "Aircraft Loss-of-Control Accident Analysis," AIAA Guidance Navigation, and Control Conference, AIAA 2010-8004, August 2010.

[4] Advani, Sunjoo and Field, Joris, "Upset Prevention and Recovery Training in Flight Simulators," AIAA Modeling and Simulation Technologies Conference, AIAA 2011-6698, August 2011

[5] Belcastro, Christine M. and Foster, John V., Shah, Gautam H., Gregory, Irene M., Cox, David E., Crider, Dennis A, Groff, Loren, Newman, Richard L.: Aircraft Loss of Control Problem Analysis and Research Toward a Holistic Solution. Journal of Guidance, Control, and Dynamics. Vol. 40, No. 4, April 2017. DOI: 10.2514/1.G002815.

[6] Cunningham, K., Shah, G. H., Frink, N. T., McMillin, S. N., Murphy, P. C., Brown, F. R., Shweyk, K. M., Nayani, S. N., "Preliminary Test Results for Stability and Control Characteristics of a Generic T-Tail Transport Airplane at High Angle of Attack," AIAA Atmospheric Flight Mechanics Conference, AIAA 2018-0629.

[7] Hyde, D. C., Brown, F. R., Shweyk, K. M., Cunningham, K., Shah, G., "High Angle of Attack Static and Dynamic Modeling Methods for Flight Dynamics Loss of Control," AIAA Atmospheric Flight Mechanics Conference, AIAA 2018-1021.

[8] Murphy, Patrick C. and Klein, Vladislav, "Validation of Methodology for Estimating Aircraft Unsteady Aerodynamic Parameters From Dynamic Wind Tunnel Tests,” AIAA Atmospheric Flight Mechanics Conference, AIAA 2003-5397, 2003.

[9] Murphy, Patrick C., Klein, Vladislav, Frink, Neal T.: Nonlinear Unsteady Aerodynamic Modeling Using Wind Tunnel and Computational Data. Journal of Aircraft. Vol. 54: 659-683, No. 2, March-April 2017. DOI: 10.2514/1.C033881.

[10] Murphy, Patrick C., Klein, Vladislav, and Frink, Neal T., "Unsteady Aerodynamic Modeling in Roll for the NASA Generic Transport Model," AIAA Atmospheric Flight Mechanics Conference, AIAA 2012-4652. 
[11] Vicroy, D. and Loeser, T., "SACCON Dynamic Wind Tunnel Tests at DNW- NWB and 14'x22' NASA LaRC", AIAA2010-4394, June 2010.

[12] Murphy, P.C., Klein, V., Frink, Neal T., and Vicroy, Dan D., "System Identification Applied to Dynamic CFD Simulation and Wind Tunnel Data," AIAA Atmospheric Flight Mechanics Conference, AIAA 2011-6522, August 2011.

[13] Frink, N. T., "Tetrahedral Unstructured Navier-Stokes Method for Turbulent Flows," AIAA Journal, Vol. 36, No. 11, November 1998, pp. 1975-1982.

[14] Frink, N. T., Pirzadeh, S. Z., Parikh, P. C., Pandya, M. J.(2000) "The NASA Tetrahedral Unstructured Software System". Aeronautical Journal, Vol. 104, No. 1040 (491-499). TetrUSS website: http://tetruss.larc.nasa.gov.

[15] Thompson, J.R., Frink, N.T., and Murphy, P. C., "Guidelines for Computing Longitudinal Dynamic Characteristics of a Subsonic Transport", AIAA Applied Aerodynamics Conference, AIAA 2010-4819, June 2010.

[16] Klein, Vladislav and Murphy, Patrick C., "Estimation of Aircraft Nonlinear Unsteady Parameters From Wind Tunnel Data," NASA TM-1998-208969, December, 1998.

[17] Klein, Vladislav and Morelli, Eugene, “Aircraft System Identification: Theory and Practice,” 1st edition, AIAA Inc., Reston, Virginia, 2006.

[18] Morelli, E. A. and Klein, V., “Aircraft System Identification: Theory and Practice," 2nd edition, Sunflyte Enterprises, Williamsburg, VA, 2016.

[19] Bommanahal, M. and Goman, M., "Nonlinear Unsteady Aerodynamic Modeling by Volterra Variational Approach,” AIAA Atmospheric Flight Mechanics Conference, AIAA 2012-4654.

\section{Figures}

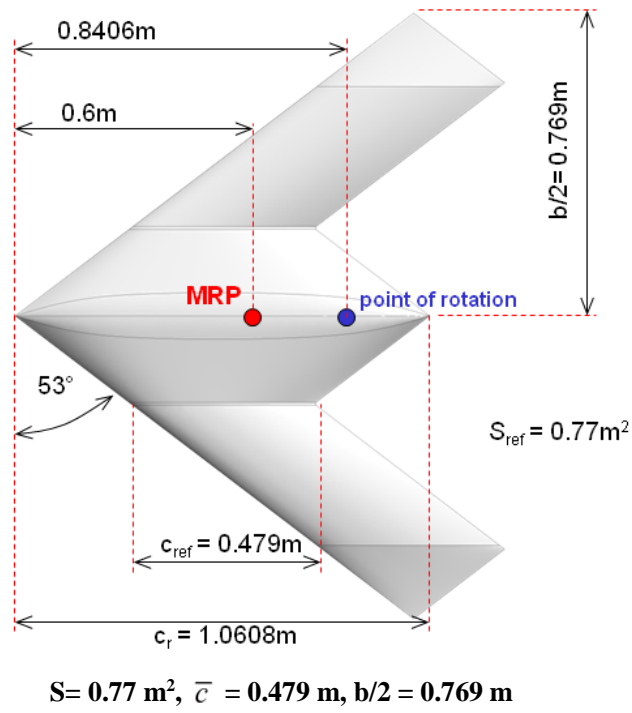

Figure 1. Model Geometry for SACCON sub-scale aircraft. 

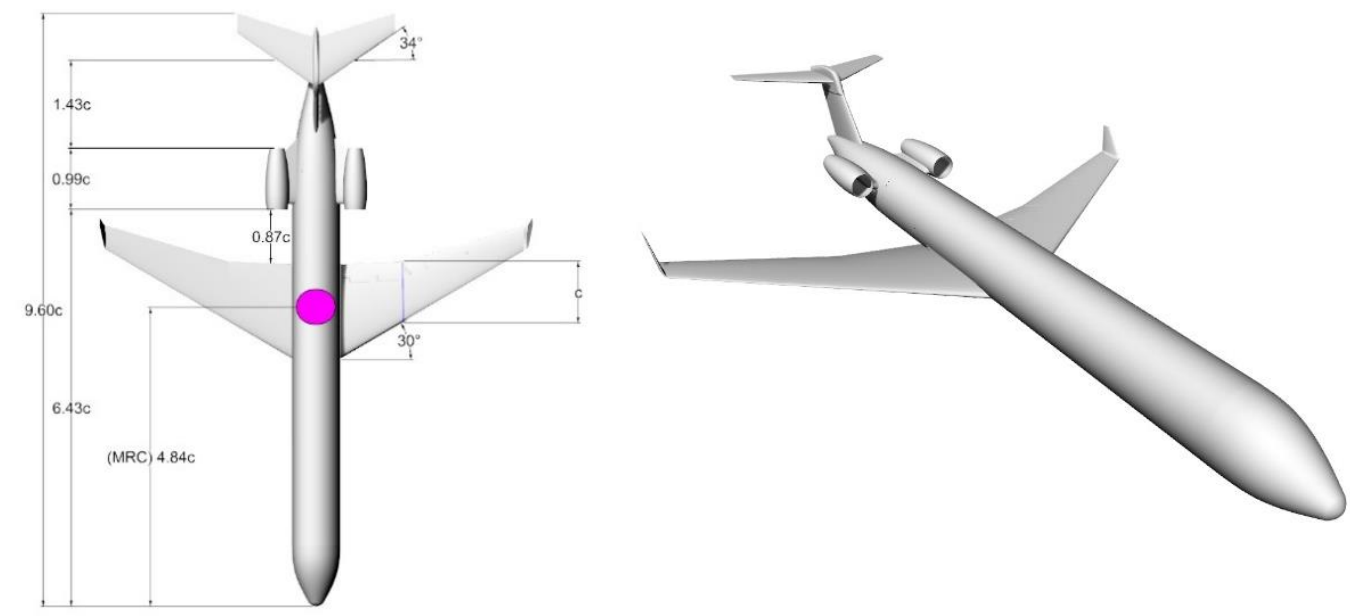

$\mathrm{S}=40.90 \mathrm{in}^{2}, \bar{c}=02.58 \mathrm{in}, \mathrm{b}=17.78 \mathrm{in}$

Figure 2. GTT model geometry and perspective view.

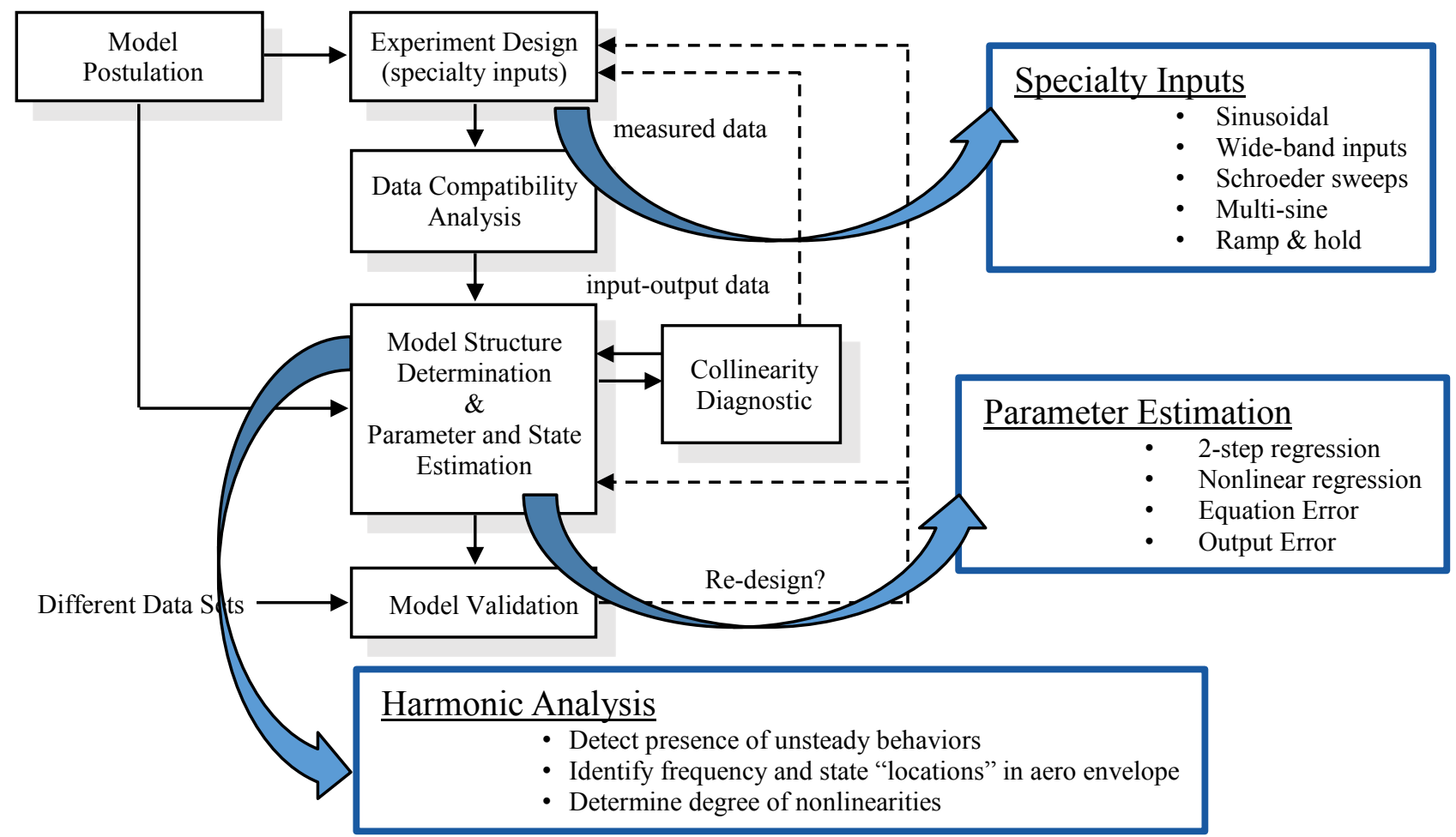

Figure 3. Aircraft System Identification for unsteady modeling. 

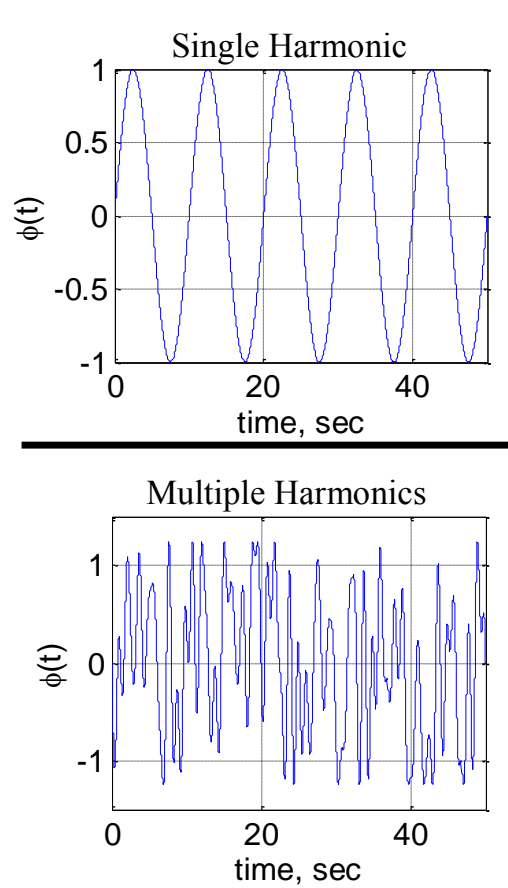
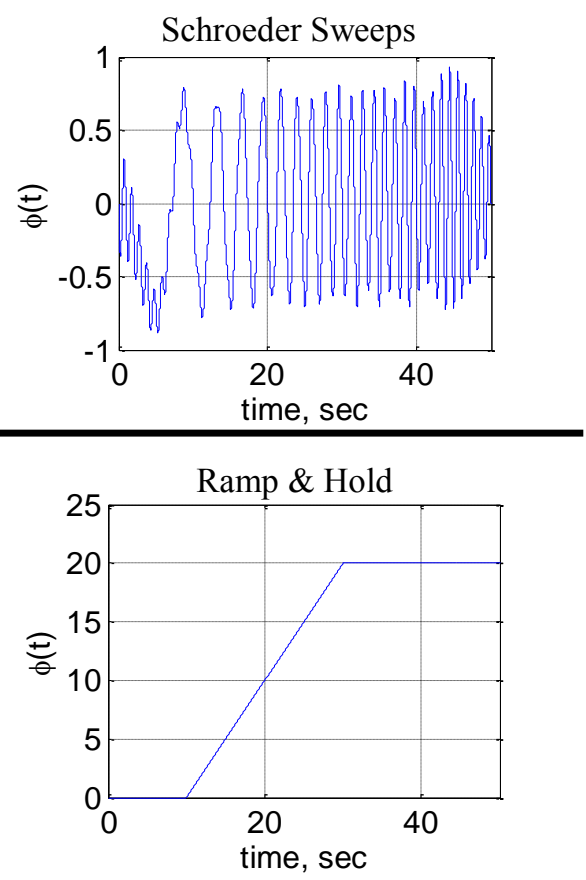

Figure 4. Candidate inputs for identification of unsteady models.

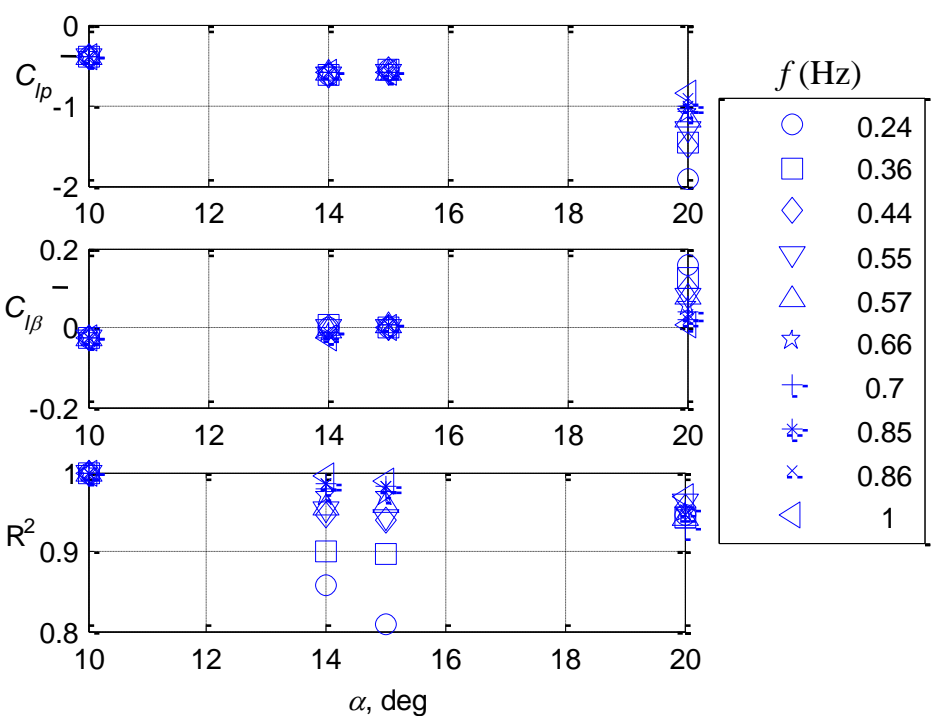

Figure 5. Roll moment out-of-phase and in-phase components from harmonic analysis of wind tunnel forced oscillations in roll, $\phi_{A}=5^{\circ}$. SACCON model. 


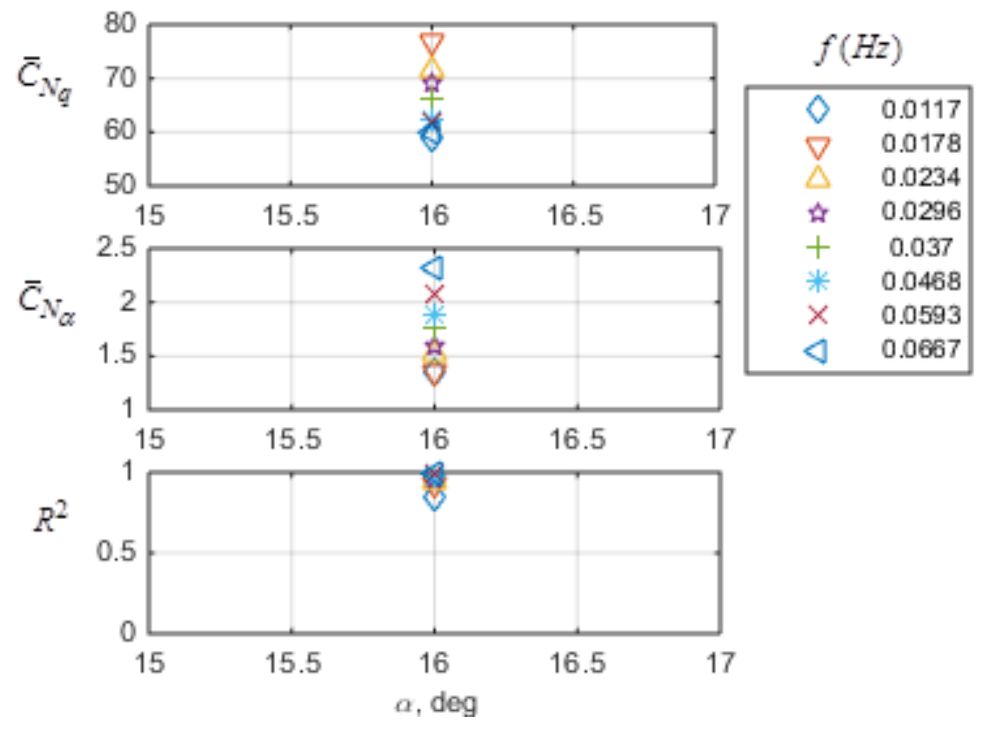

Figure 6a. Harmonic analysis of $C_{N}$ using first-order model and data from water tunnel forced oscillations in pitch, $\alpha_{A}=5^{\circ}, \alpha_{0}=16^{\circ}$. GTT model.
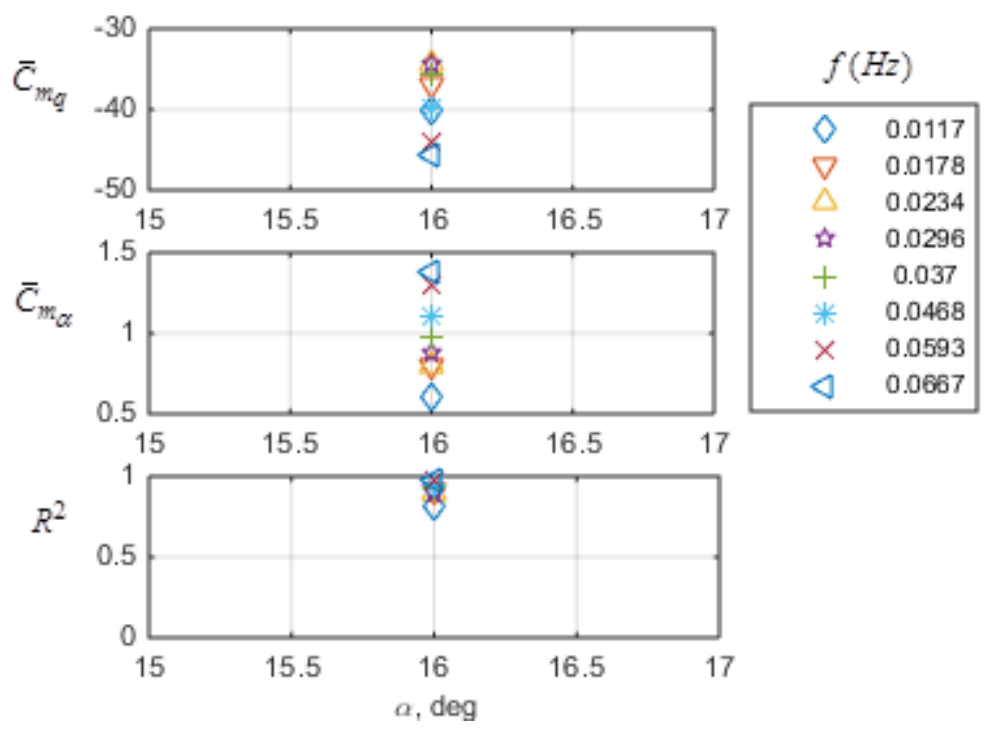

Figure $6 \mathrm{~b}$. Harmonic analysis of $\boldsymbol{C}_{\boldsymbol{m}}$ using first-order model and data from water tunnel forced oscillations in pitch, $\alpha_{A}=5^{\circ}, \alpha_{0}=16^{\circ}$. GTT model. 

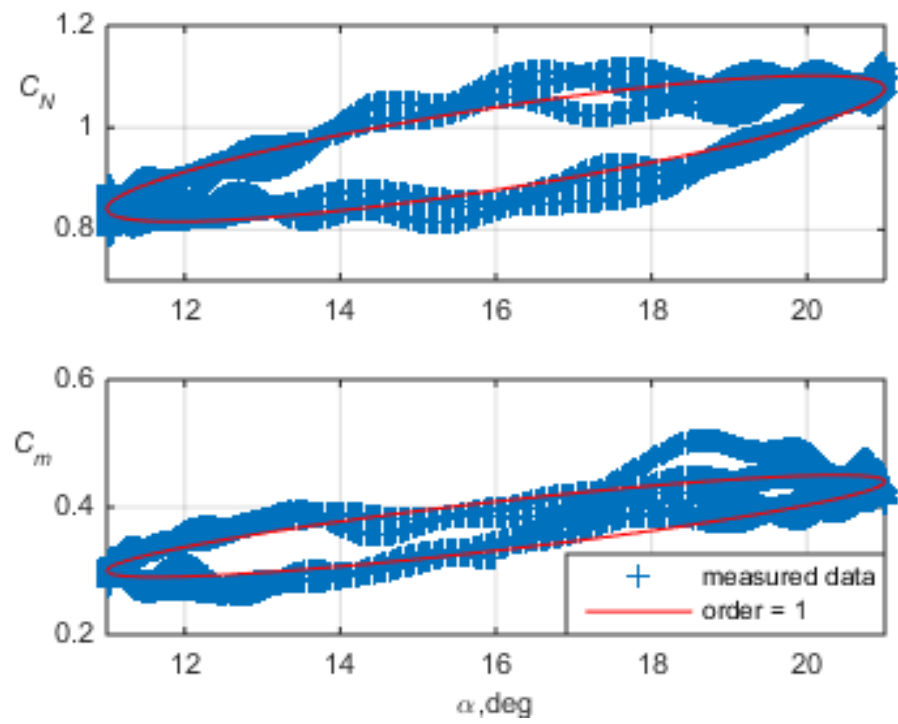

Figure 7. First-order harmonic model and water-tunnel measurements for $C_{N}$ and $C_{m}$ at $\mathrm{k}=\mathbf{0 . 0 1 2}$, GTT model.
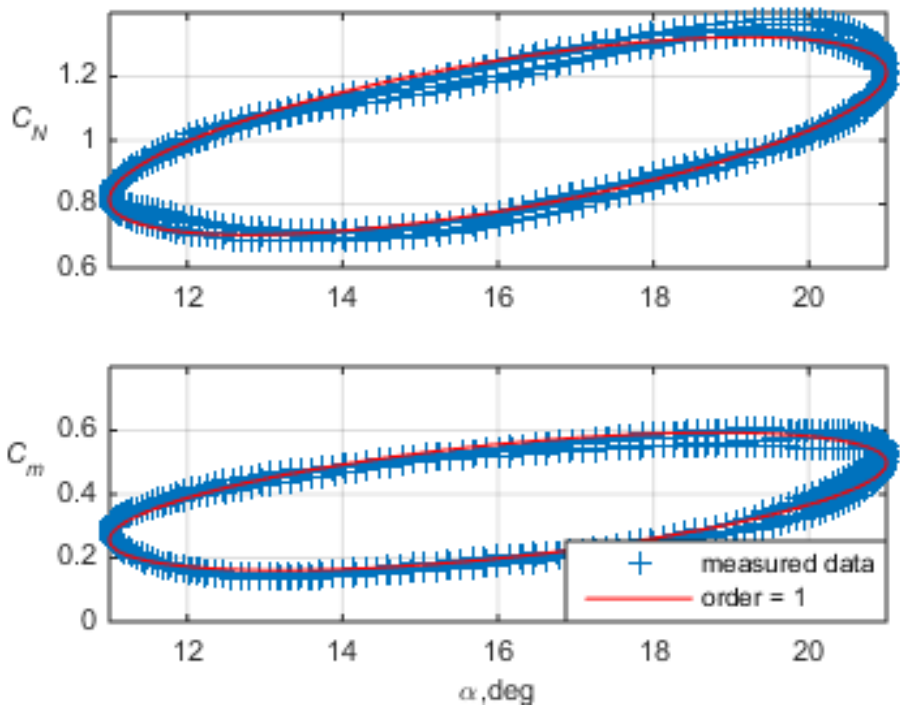

Figure 8. First-order harmonic model and water-tunnel measurements for $C_{N}$ and $C_{m}$ at $\mathrm{k}=\mathbf{0 . 0 4 5}$. GTT model. 

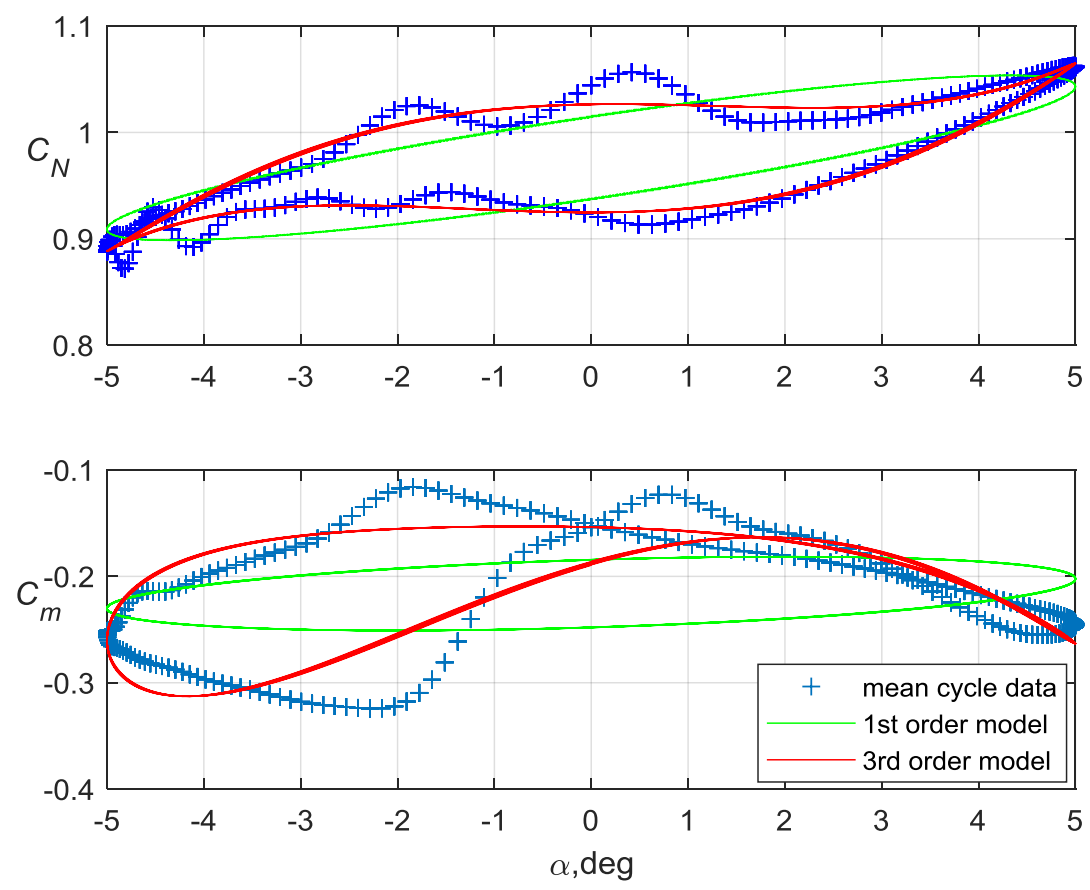

Figure 9. First and Third-order harmonic model and CFD measurements for $C_{N}$ and $C_{m}$ at $k=0.0079$, GTT model.
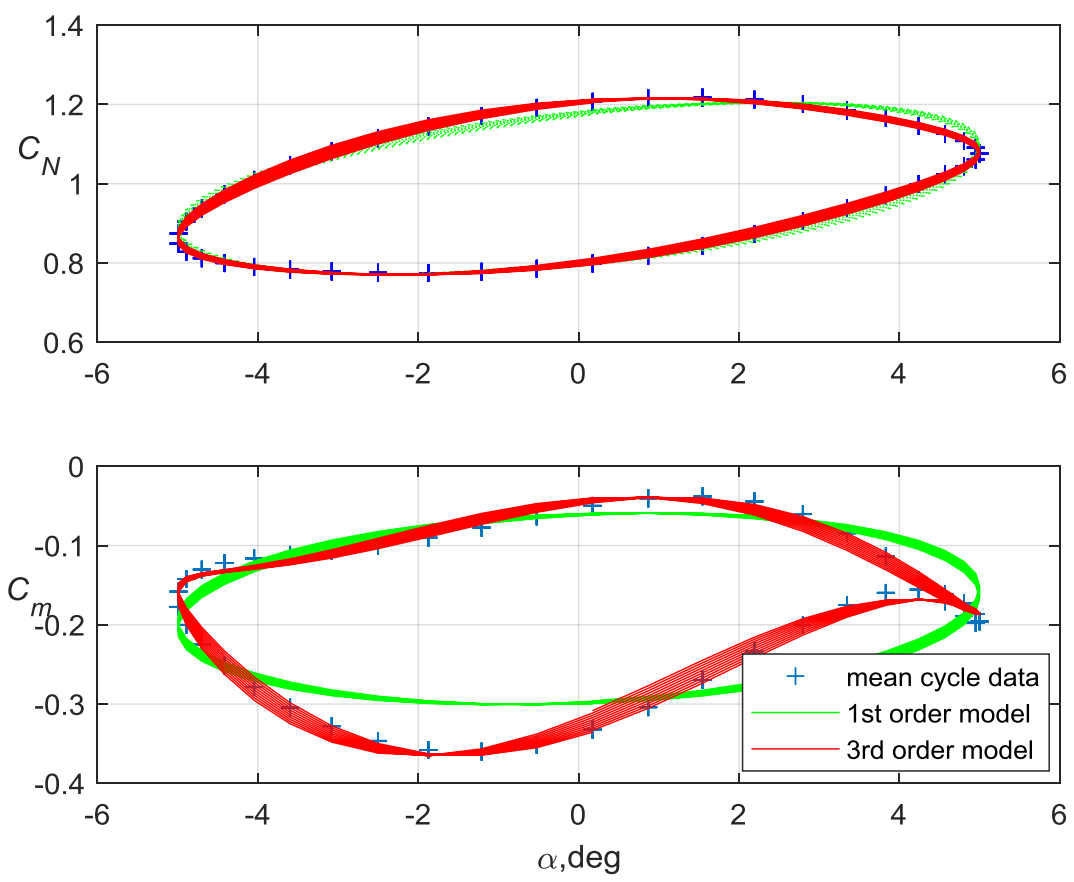

Figure 10. First and Third-order harmonic model and CFD measurements for $C_{N}$ and $C_{m}$ at $k=0.0450$, GTT model. 

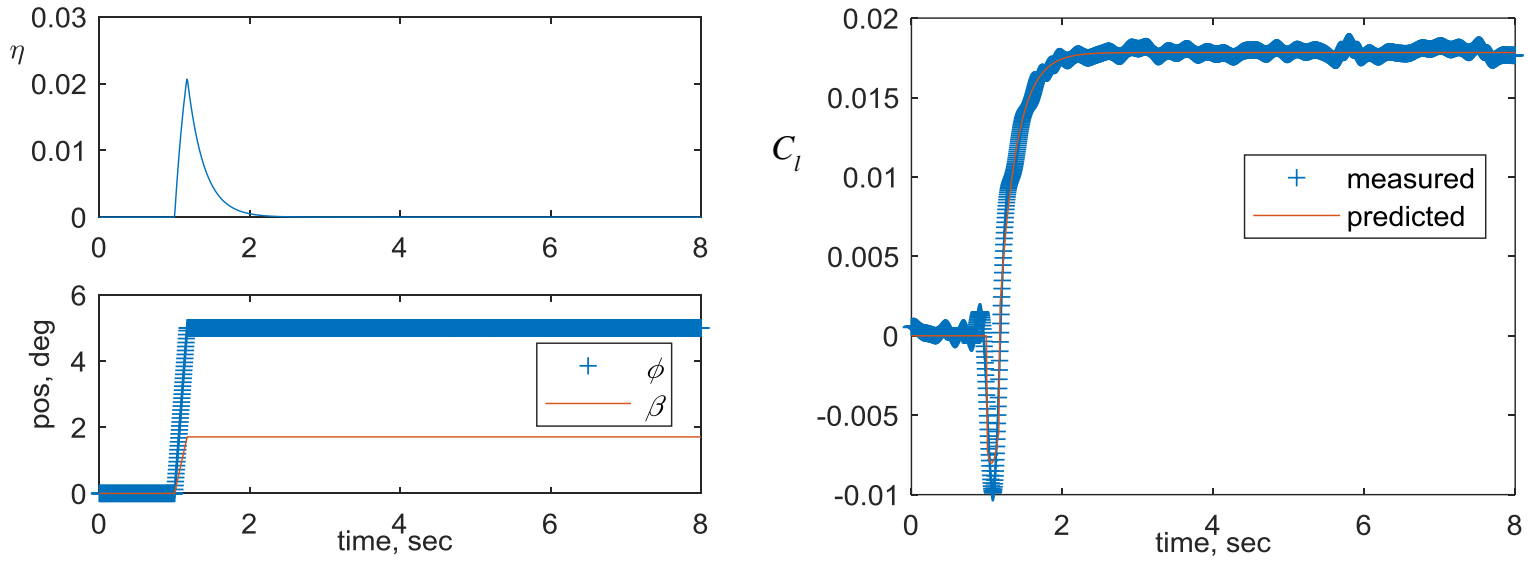

Figure 11. For simulated SACCON test, measured and predicted linear unsteady responses in $C_{l}$ to $30 \mathrm{deg} / \mathrm{sec}$ ramp at $\alpha_{0}=20^{\circ}$.

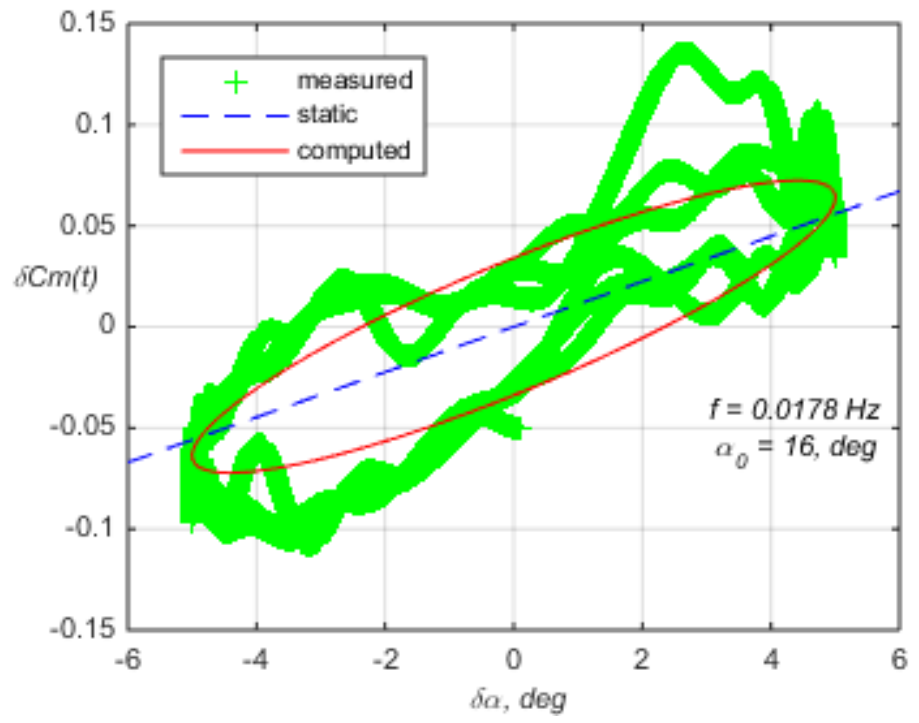

Figure 12. Linear unsteady model and water-tunnel measurements for $C_{m}$ at $\mathrm{k}=0.012$, and $\alpha_{0}=16^{\circ}$, GTT model. 


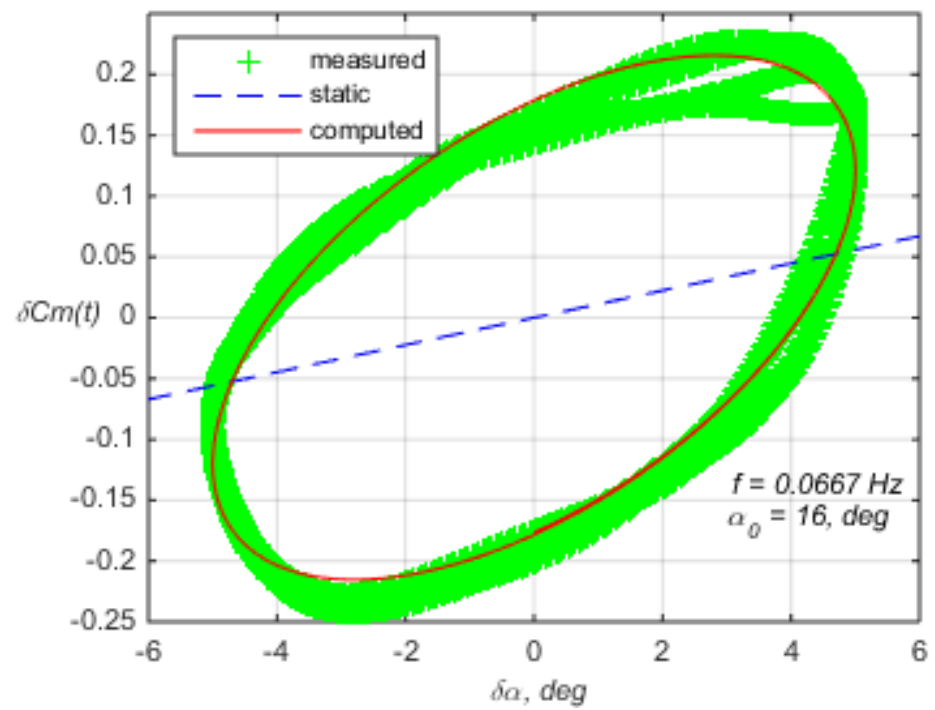

Figure 13. Linear unsteady model and water-tunnel measurements for $C_{m}$ at $\mathrm{k}=0.045$, and $\alpha_{0}=16^{\circ}$, GTT model.

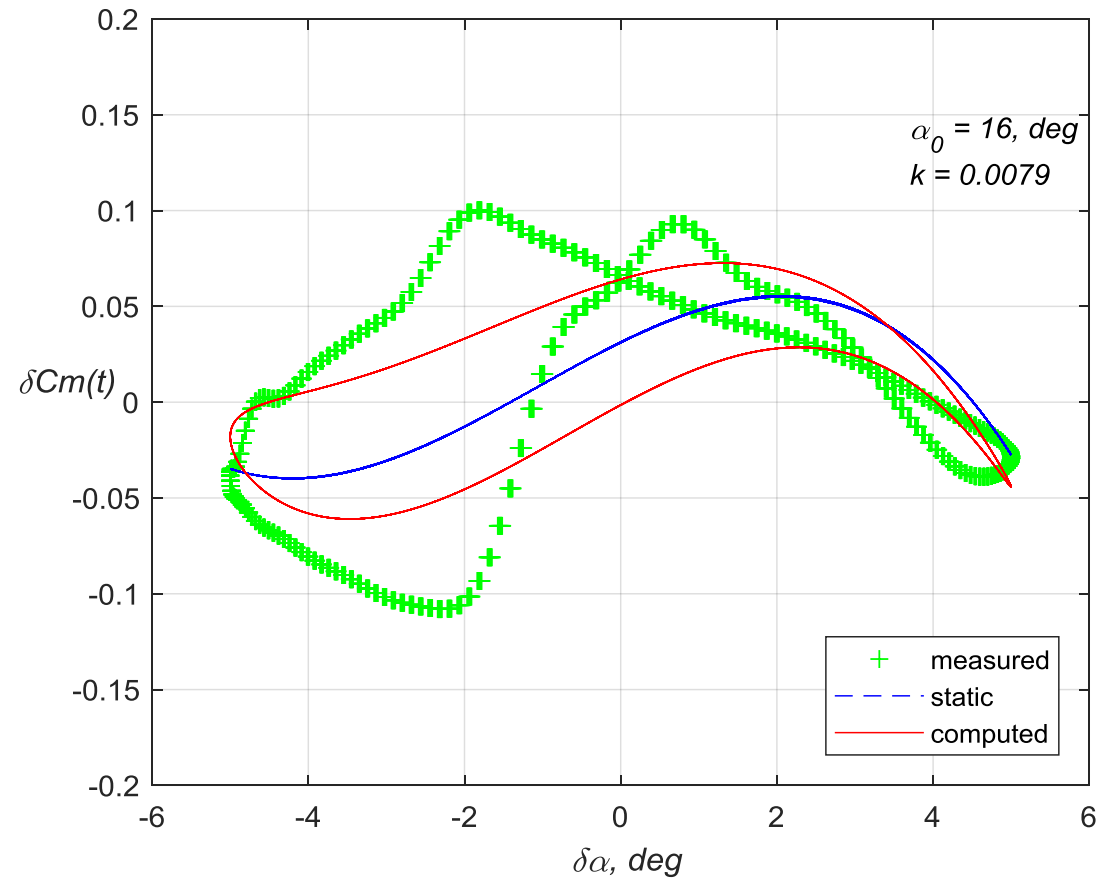

Figure 14. Third-order unsteady model and CFD "measurements" for $C_{m}$ at $k=0.0079$, GTT model, sinusoidal inputs. 


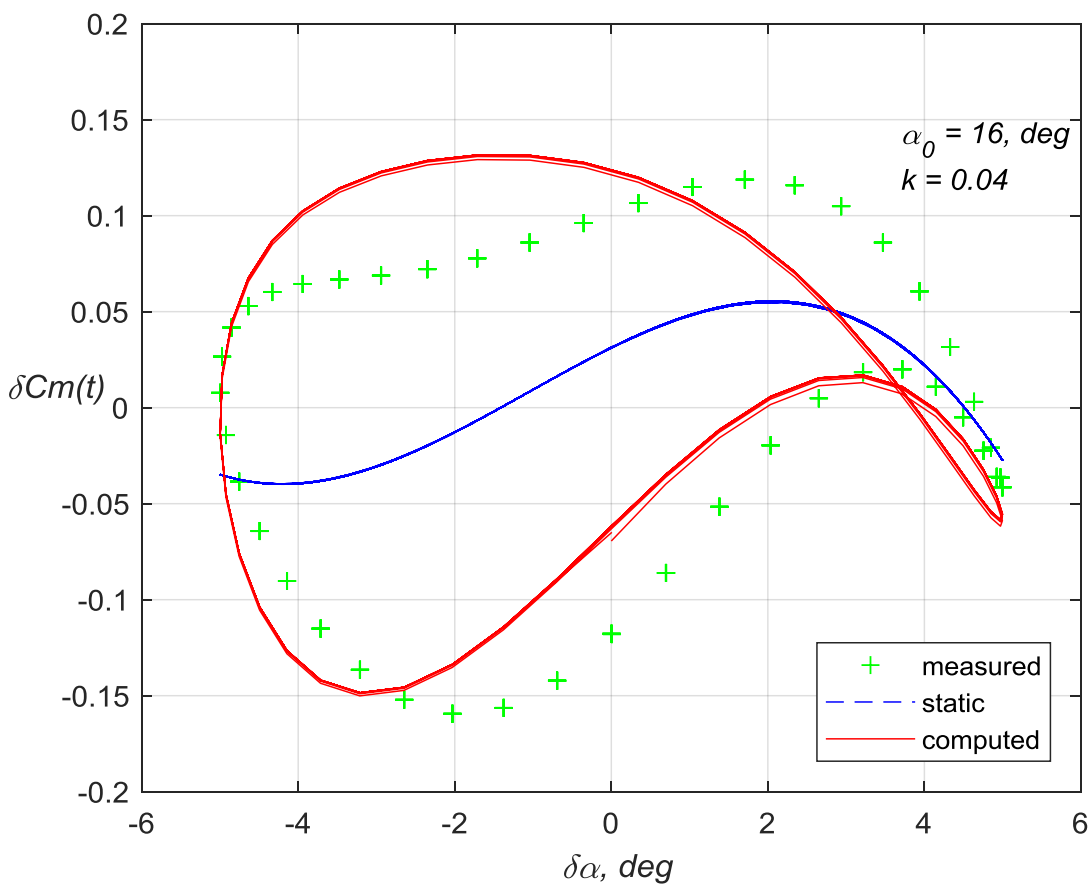

Figure 15. Third-order unsteady model and CFD "measurements" for $C_{m}$ at $k=0.0400$, GTT model, sinusoidal inputs.

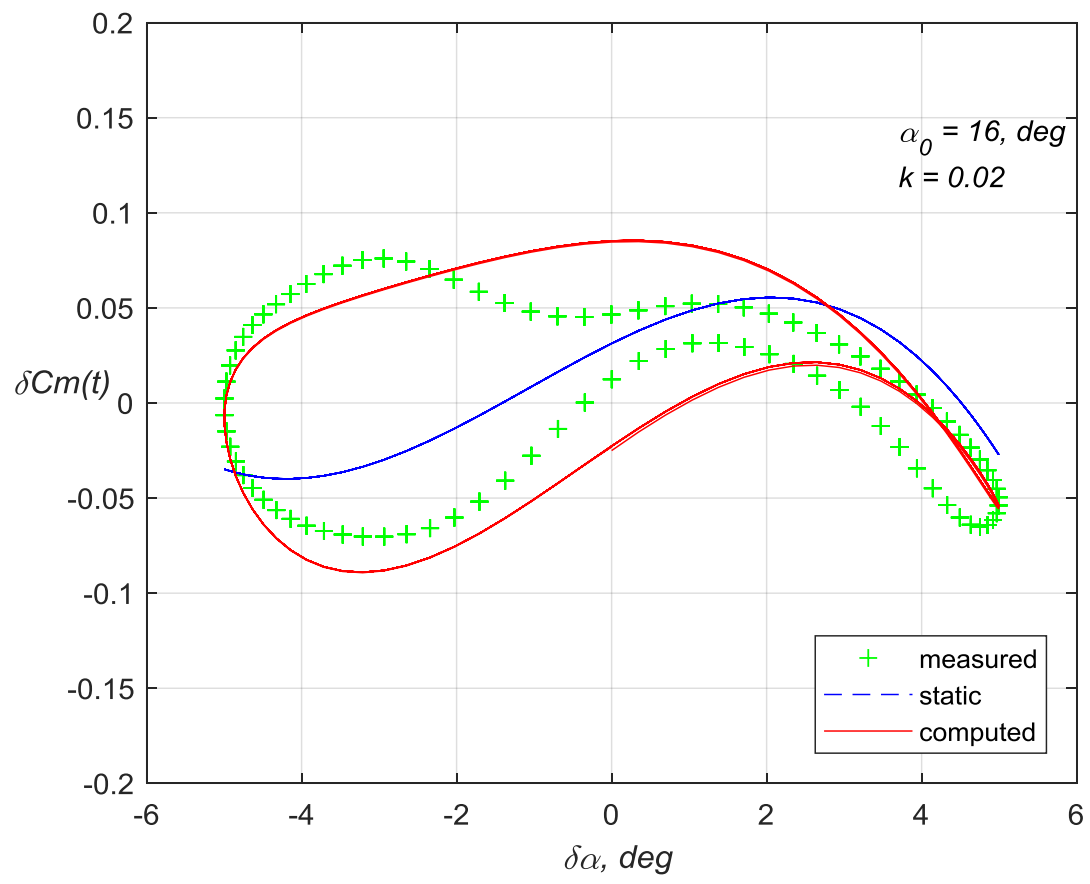

Figure 16. Validation Test. Third-order unsteady model and CFD "measurements" for $C_{m}$ at $\boldsymbol{k}=0.0200$, GTT model, sinusoidal inputs. 

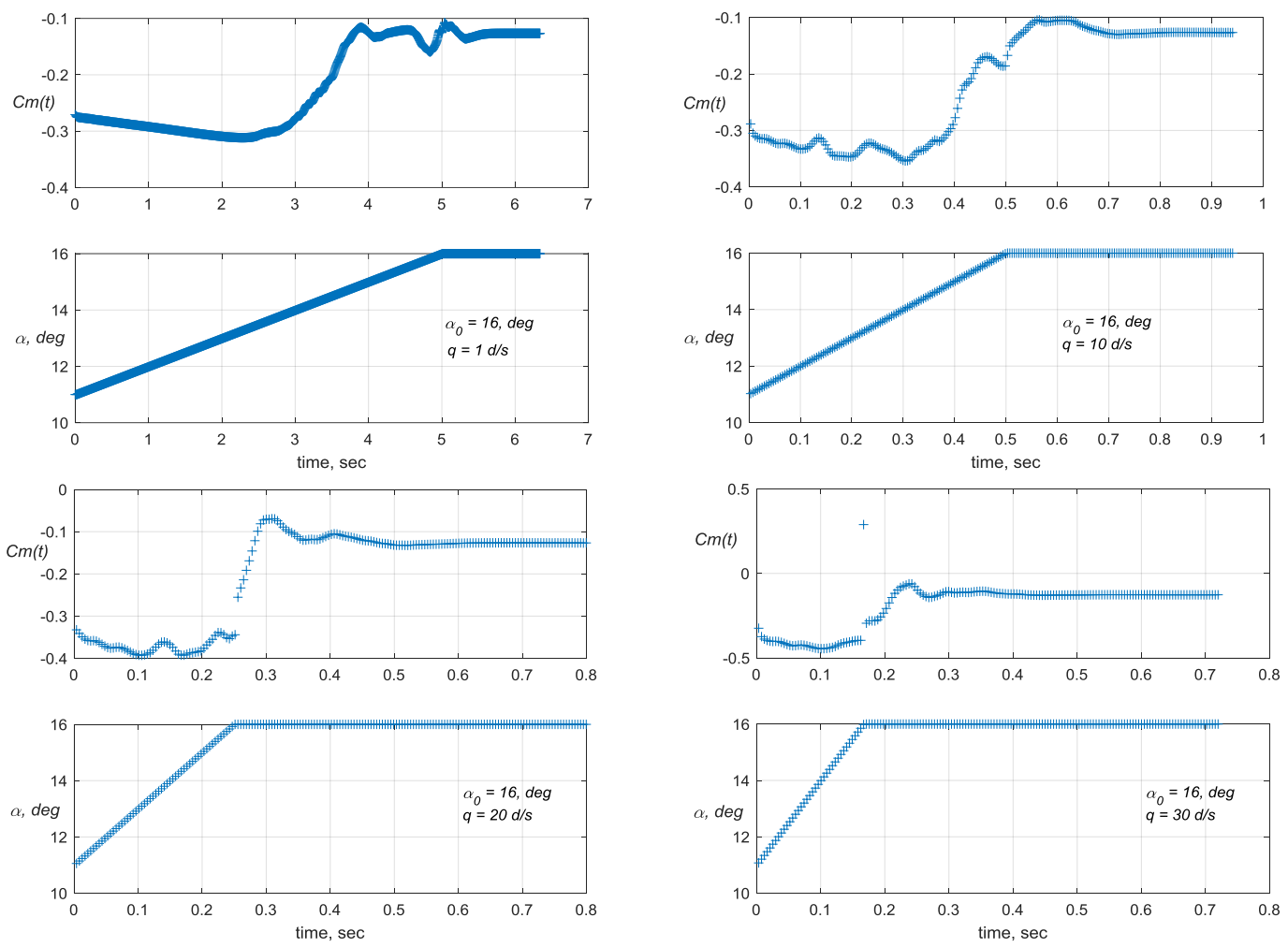

Figure 17. CFD simulated $C_{m}$ ramp "measurements" for GTT model.
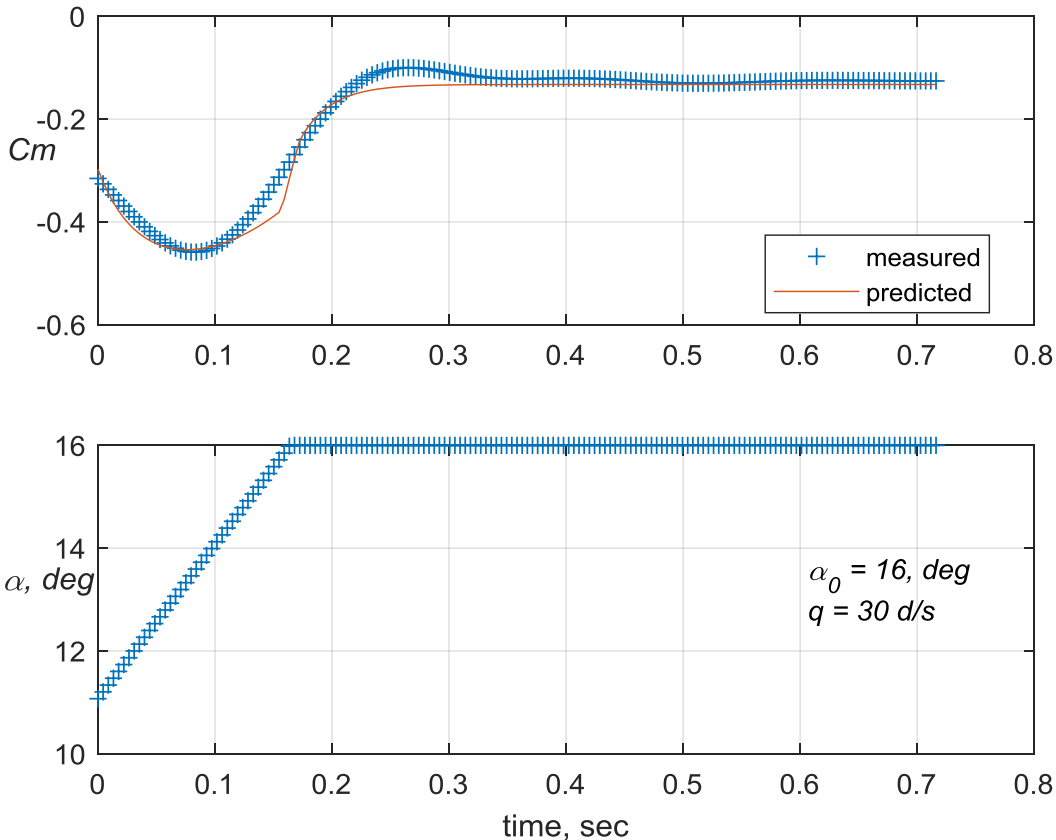

Figure 18. Example unsteady model from CFD "measurements" for $C_{m}$ under $30 \mathrm{~d} / \mathrm{s} \mathrm{ramp}$, GTT model. 